\title{
On certain weak phase transformations in multilattices
}

\author{
Mario Pitteri \\ DMMMSA, Università di Padova \\ Via Belzoni 7, 35131 Padova ITALY \\ email: pitteri@dmsa.unipd.it *
}

\begin{abstract}
This paper is dedicated to the memory of Clifford Truesdell, to whom I acknowledge my debt and gratitude. I present some recent results of mine on thermomechanics of crystalline solids, a research on which I began working during my stay at The Johns Hopkins University, 1977-1979. Then, in the last section, I mention some unusual merits of Truesdell, which I experienced in my scientific career and are not widely known.
\end{abstract}

Keywords Phase transformations, thermoelasticity, crystalline solids, quartz, physical possibility.

\section{Introduction}

I have many reasons to be grateful to Clifford Truesdell, both as a scientist and as a person. Along my research activity as a postdoc I soon realized that I needed to deepen my knowledge of kinetic-statistical theories of mechanics, and The Johns Hopkins University was my choice because of Truesdell's work on the kinetic theory of gases - he was then carrying to completion his book [50]. During my stay there I have also been exposed to other subjects I was not so familiar with, among which elements of experimental continuum mechanics and of the history of mechanics, classical thermodynamics along Carnot's lines, stability problems in continuum mechanics, and nonlinear elasticity of crystals. In fact, in the past fifteen years the last topic has been the main focus of my research, the most recent results of which constitute the core of this paper.

There has recently been a renewed interest in the geometry and kinematics of multilattices, in view of constructing a nonlinear model of the thermomechanical behavior of complex crystals. Background, some details and references

*I want to extend here to Charlotte Truesdell my gratitude to her husband Clifford expressed below. This work is part of the research activities of the EU Network 'Phase Transitions in Crystalline Solids', and is partially supported by the Italian M.U.R.S.T. through the project 'Mathematical Models for Materials Science'. 
are given in [44], where also some still unsolved problems are outlined. One of these is the formulation of a unified kinematics of multilattices of different complexity. Indeed, any multilattice configuration admits a maximal skeletal lattice of translations mapping the multilattice to itself (the essential skeleton), which nevertheless shares the property of being translation-invariant with any one of its infinitely many sublattices. Once a sublattice of the essential skeleton is selected, additional vectors (shifts) have to be introduced to describe the multilattice configuration, and these change, possibly also in number, with the changing sublattice. The kinematics developed so far works well for multilattices whose lattice of translations is the essential skeleton itself; these will be called essentially described, or essential for short. In this paper we restrict the attention to essential multilattices alone. Further work is needed to cope with deformations along which a multilattice changes its periodicity, in a suitable sense. ${ }^{1}$ Handling such deformations would be of great interest from the theoretical point of view as well as for the applications, for instance to certain phase transitions of shape memory alloys.

In [43] it is shown by a simple example how the knowledge of the full geometry and kinematics of essential multilattices can help in classifying all the possibilities for their weak - that is, involving suitably small distortions - symmetrybreaking thermoelastic phase transformations, and motivation for the procedure is provided. The example is the diatomic 2-lattice used in the introductory sections of [47] to describe what is called a structural phase transition in a crystalline solid: in a primitive tetragonal lattice whose unit cell has a physically different atom in its center the transition is driven by the central atom moving off center. That this can happen in essentially two different ways is shown to be one of the possibilities; the other, orthogonal to the first in a suitable sense, corresponds to a configurational transition: the tetragonal skeleton drives the transformation, which is then accompanied by a suitable displacement of the central atom. For this kind of transition the independent generic possibilities have been classified in [23].

Here, in $\S 3$, I provide an explicit, general framework for generic weak bifurcations in essential multilattices, completing a scheme presented in [24]. As an example, in $\S 4$ we study the structural transitions of $\beta$-quartz, described by the 3-lattice model introduced in [36]. The analysis shows that, among others, there are two - mutually orthogonal in a sense, and both described by a 1-dimensional order parameter space - trigonal trapezohedral low-symmetry product phases, one of which is the $\alpha$-phase as modelled in [36]. The other was obtained in [24] as an outcome of the search for a third quartz phase, used to give an alternative to the so-called incommensurate phase introduced in the physical literature to explain certain peculiarities of the $\alpha-\beta$ transition. Here the geometry of the 3-lattice describing this new phase is given in detail.

Finally, since this paper is appearing in a volume dedicated to the memory of

\footnotetext{
${ }^{1}$ An example is a controlled deformation of a body-center cubic (bcc) simple lattice along which the central atom starts moving off-center for certain (transition) values of the controls. At the transition the nonessential 2-lattice equivalent to the essential bcc simple lattice starts deforming into an essential 2-lattice.
} 


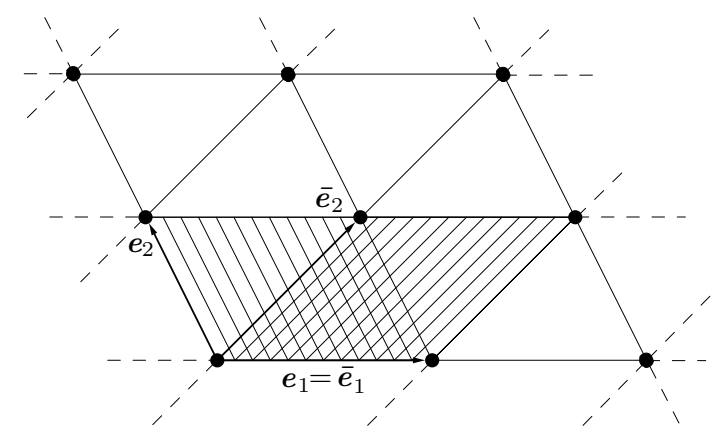

Figure 1: Equivalent bases for a given (planar) lattice

Clifford Truesdell, in $\S 5$ I detail some unusual merits of him, which I experienced in my scientific career and are not widely known.

\section{Preliminaries}

I sketch here the bare essentials for the rest of the paper, addressing the reader to [44] for more information. In particular, as there, I use the summation convention and 'running indices' without specifying their range; for instance in expressions like 'the lattice basis $\boldsymbol{e}_{a}$ ' instead of 'the lattice basis $\left\{\boldsymbol{e}_{a}, a=1,2,3\right\}$ ', or 'the function $\hat{\phi}\left(\boldsymbol{e}_{a}, \boldsymbol{p}_{r}, \theta\right)$ ' instead of 'the function $\hat{\phi}\left(\boldsymbol{e}_{1}, \boldsymbol{e}_{2}, \boldsymbol{e}_{3}, \boldsymbol{p}_{1}, \ldots, \boldsymbol{p}_{n-1}, \theta\right)$ '. This should not generate too much confusion. Also, the relations $\leq$ and $<$ between groups mean subgroup of and proper subgroup of, respectively.

Let $\mathbb{Z}$ and $\mathbb{R}$ denote the integral and real numbers, respectively. Consider first the simplest triply-periodic structures, that is, simple lattices (or 1-lattices):

$$
\mathcal{L}=\left\{N^{a} \boldsymbol{e}_{a}, a=1,2,3, N^{a} \in \mathbb{Z}\right\}=\mathcal{L}\left(\boldsymbol{e}_{a}\right) .
$$

The lattice vectors (or lattice basis) $\boldsymbol{e}_{a}$ are linearly independent in $\mathbb{R}^{3}$.

Any basis $\boldsymbol{e}_{a}$ uniquely determines the 1-lattice $\mathcal{L}\left(\boldsymbol{e}_{a}\right)$, but not vice versa:

$$
\mathcal{L}\left(\overline{\boldsymbol{e}}_{a}\right)=\mathcal{L}\left(\boldsymbol{e}_{a}\right) \Leftrightarrow \overline{\boldsymbol{e}}_{a}=m_{a}^{b} \boldsymbol{e}_{b}, \quad m \in G L(3, \mathbb{Z}) ;
$$

here $G L(3, \mathbb{Z})$ is the group of 3 by 3 integral matrices with determinant \pm 1 . The crystallographic point group (or holohedry), $P\left(\boldsymbol{e}_{a}\right)$, of the lattice $\mathcal{L}\left(\boldsymbol{e}_{a}\right)$ is then defined as the group of all the orthogonal transformations mapping $\mathcal{L}\left(\boldsymbol{e}_{a}\right)$ to itself; equivalently:

$$
P\left(\boldsymbol{e}_{a}\right)=\left\{\boldsymbol{Q} \in O(3): \boldsymbol{Q} \boldsymbol{e}_{a}=m_{a}^{b} \boldsymbol{e}_{b}\right\} .
$$

Notice that the basis $\boldsymbol{e}_{a}$ satisfies (3) if and only if the lattice metric

$$
C=\left(C_{a b}\right), \quad C_{a b}=e_{a} \cdot e_{b},
$$



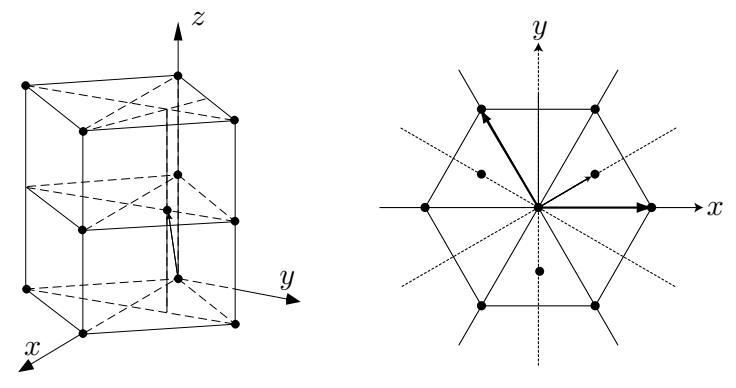

Figure 2: An elementary cell for the hcp lattice, and its projection on the $x-y$ basal plane

is a fixed point of the map

$$
C \mapsto m^{t} C m .
$$

The conjugacy classes of the holohedries in $O(3)$ correspond to the well known 7 crystal systems.

By looking at the right-hand side of the equation defining $P\left(\boldsymbol{e}_{a}\right)$ in (3) we introduce the lattice group $L\left(\boldsymbol{e}_{a}\right)$ of a lattice $\mathcal{L}\left(\boldsymbol{e}_{a}\right)$, associated with its lattice basis $\boldsymbol{e}_{a}$ :

$$
\begin{aligned}
L\left(\boldsymbol{e}_{a}\right) & =\left\{m \in G L(3, \mathbb{Z}): m_{a}^{b} \boldsymbol{e}_{b}=\boldsymbol{Q} \boldsymbol{e}_{a}, \boldsymbol{Q} \in P\left(\boldsymbol{e}_{a}\right)\right\} \\
& =\left\{m \in G L(3, \mathbb{Z}): m^{t} C m=C\right\}
\end{aligned}
$$

By the last equality the lattice group depends on the basis $\boldsymbol{e}_{a}$ only through the corresponding metric $C$, hence can be denoted by $L(C)$. The conjugacy classes of lattice groups in $G L(3, \mathbb{Z})$ correspond to the well known 14 (Bravais) lattice types.

Real crystals (hexagonal metals, alloys, etc.) are not in general 1-lattices. Their geometry and kinematics can be described by means of multilattices, which are the union of a finite number of nontrivial translates of a 1-lattice. A simple, well known example of 2-lattice is the hexagonal close-packed (hcp) structure, which is sketched in Fig. 2.

In general, an $n$-lattice $\mathcal{M}$ in 3-dimensional affine space can be defined as follows, by choosing the origin $O$ at one of the lattice points and setting $\boldsymbol{p}_{0}=\mathbf{0}$ for convenience:

$$
\mathcal{M}=\mathcal{M}\left(\boldsymbol{e}_{a}, \boldsymbol{p}_{1}, \ldots, \boldsymbol{p}_{n-1}\right)=\cup_{r=0}^{n-1}\left\{O+\mathcal{L}\left(\boldsymbol{e}_{a}\right)+\boldsymbol{p}_{r}\right) ;
$$

$\mathcal{L}\left(\boldsymbol{e}_{a}\right)$ is called the skeletal lattice of $\mathcal{M}$. Fig. 3 is a schematic picture of a 2-dimensional (planar) 2-lattice; if the atoms represented by filled circles are phisically indistinguishable from the ones represented by open circles, the 2lattice is called monatomic, otherwise diatomic. 


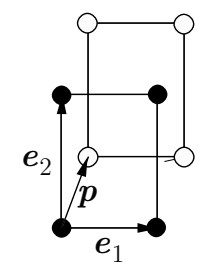

Figure 3: Unit cells of the component 1-lattices of a planar 2-lattice

The multilattice descriptors $\left(\boldsymbol{e}_{a}, \boldsymbol{p}_{r}\right)=: \boldsymbol{\varepsilon}_{\sigma}, \sigma=1, \ldots, n+2$, (in terms of which we can write $\left.\mathcal{M}=\mathcal{M}\left(\varepsilon_{\sigma}\right)\right)$ satisfy the following conditions guaranteeing the three-dimensionality of $\mathcal{M}$ and the non-overlap of the constituent 1-lattices:

$$
\boldsymbol{e}_{1} \cdot \boldsymbol{e}_{2} \times \boldsymbol{e}_{3} \neq 0, \quad \boldsymbol{p}_{r} \neq \boldsymbol{p}_{s}+l_{r s}^{a} \boldsymbol{e}_{a}, \quad r, s=0, \ldots, n-1, l_{r s}^{a} \in \mathbb{Z},
$$

with the exclusion of the case $r=0=s$.

An $n$-lattice $\mathcal{M}$ is called essential if its skeletal lattice contains all the translations mapping $\mathcal{M}$ to itself. In this case the lattice cell has minimum volume.

The $O(3)$-invariant multilattice metric

$$
K=\left(K_{\sigma \tau}\right), \quad K_{\sigma \tau}=K_{\tau \sigma}=\varepsilon_{\sigma} \cdot \varepsilon_{\tau}, \quad \sigma, \tau=1, \ldots, n+2,
$$

is an analog of (4). The manifold $\mathcal{Q}_{n+2}^{m}$ of multilattice metrics is a submanifold of the vector space $\mathcal{Q}_{n+2}$ of all symmetric matrices in $\mathbb{R}^{n+2} ; \mathcal{Q}_{n+2}^{m}$ is a 'state space' for $n$-lattices analogous to the set $\mathcal{Q}_{3}^{>}$of positive definite quadratic forms in $\mathbb{R}^{3}$ (the lattice metrics) for 1-lattices.

The 'global symmetry group' of essential $n$-lattices expresses the indeterminateness in the choice of the multilattice descriptors:

$$
\mathcal{M}\left(\bar{\varepsilon}_{\sigma}\right)=\mathcal{M}\left(\varepsilon_{\sigma}\right) \Leftrightarrow \bar{\varepsilon}_{\sigma}=\mu_{\sigma}^{\tau} \varepsilon_{\sigma}, \quad \mu \in \Gamma_{n+2},
$$

where $\Gamma_{n+2}<G L(n+2, \mathbb{Z})$ consists of the matrices of the form

$$
\left(\mu_{\sigma}^{\tau}\right)=\left(\begin{array}{ccc|c}
m_{a}^{b} & l_{1}^{b} \cdots l_{n-1}^{b} \\
\hline 0 & 0 & 0 & \\
& \vdots & & \alpha_{r}^{s} \\
0 & 0 & 0 &
\end{array}\right), \quad \bar{\alpha}=\left(\begin{array}{ccccc}
1 & 0 & \cdots & 0 & 0 \\
0 & 1 & \cdots & 0 & 0 \\
\vdots & \vdots & \cdots & \vdots & \vdots \\
-1 & -1 & \cdots & -1 & -1 \\
\vdots & \vdots & \cdots & \vdots & \vdots \\
0 & 0 & \cdots & 1 & 0 \\
0 & 0 & \cdots & 0 & 1
\end{array}\right),
$$

for $a, b=1,2,3, r, s=1, \ldots, n-1,\left(m_{a}^{b}\right) \in G L(3, \mathbb{Z}), l_{r}^{b} \in \mathbb{Z}$, and $\alpha=\left(\alpha_{r}^{s}\right)$ belonging to the finite non-commutative group of matrices generated by the permutation matrices of the set $\{1, \ldots, n-1\}$ and by the $n-1$ by $n-1$ matrices $\bar{\alpha}$ of the form $(11)_{2}$, which are obtained from the identity by replacing one of its rows by a row of -1 s. If $\alpha$ is not a permutation of $\{1, \ldots, n-1\}$, then necessarily one of its rows consists of -1 s. 
The group $\Gamma_{n+2}$ acts in a natural way, analogous to (5), on the manifold of multilattice metrics:

$$
K \mapsto \mu^{t} K \mu .
$$

Among all changes of descriptors, particular importance have those which produce an affine isometry of the multilattice onto itself. If, with respect to the chosen origin $O$ (which is one of the lattice points) we represent the isometry as a pair $(\boldsymbol{t}, \boldsymbol{Q}), \boldsymbol{t} \in \mathbb{R}^{3}, \boldsymbol{Q} \in O(3)$, it must be, for $k=0$ if $\alpha$ is a permutation or, otherwise, for the index $k$ of the row of $\alpha$ made of -1 s - see (11):

$$
\boldsymbol{Q} \boldsymbol{\varepsilon}_{\sigma}=\mu_{\sigma}^{\tau} \varepsilon_{\tau}, \quad \boldsymbol{t}=\boldsymbol{p}_{k}+n_{0}^{a} \boldsymbol{e}_{a}, n_{0}^{a} \in \mathbb{Z}
$$

In terms of the metric $K$ in (9) equality $(13)_{1}$ is equivalent to the condition that $K$ be a fixed point for the corresponding map (12):

$$
\mu^{t} K \mu=K
$$

For any essentially described multilattice $\mathcal{M}=\mathcal{M}\left(\varepsilon_{\sigma}\right)$ the solutions $(\boldsymbol{t}, \boldsymbol{Q})$ of (13) depend on $\mathcal{M}$ itself and not on its specific descriptors $\varepsilon_{\sigma}$, and constitute the space group $S(\mathcal{M})$ of $\mathcal{M}$. The orthogonal maps appearing in $(13)_{1}$ constitute the crystal class $P(\mathcal{M})$ (or $P\left(\varepsilon_{\sigma}\right)$ ) of $\mathcal{M}$, which is a subgroup of the skeletal holohedry $P\left(\boldsymbol{e}_{a}\right)$. The matrices $\mu \in \Gamma_{n+2}$ in $(13)_{1}$ form the lattice group $\Lambda\left(\varepsilon_{\sigma}\right)<$ $G L(n+2, \mathbb{Z})$ of $\mathcal{M}\left(\varepsilon_{\sigma}\right)$. The finite group $\Lambda\left(\varepsilon_{\sigma}\right)$ depends on the choice of the $\varepsilon_{\sigma}$ - actually, on the corresponding metric $K$, so that it can be denoted by $\Lambda(K)$ and, under a change of descriptors, changes to a conjugate group in $\Gamma_{n+2}$. The conjugacy classes of lattice groups in $\Gamma_{n+2}$ formalize the notion of (arithmetic) $n$-lattice types, and the way $\Gamma_{n+2}$ acts on the state space $\mathcal{Q}_{n+2}^{m}$ gives information on the kinematics of deformable $n$-lattices. This analysis is considerably simpler if one restricts the attention to suitably small distortions:

Proposition 1 Any multilattice metric $K$ has a $\Lambda(K)$-invariant neighborhood $\mathcal{N}$ in $\mathcal{Q}_{n+2}^{m}$, to be called a wt-nbhd of $K$, such that, for any $\mu \in \Gamma_{n+2}$,

$$
\mu^{t} \mathcal{N} \mu \cap \mathcal{N} \neq \emptyset \Leftrightarrow \mu \in \Lambda(K) \Leftrightarrow \mu^{t} K \mu=K
$$

Therefore, in any $\Lambda(K)$-invariant neighborhood of $K$ contained in $\mathcal{N}$ the global $\Gamma_{n+2}$-invariance reduces to the invariance under the lattice group $\Lambda(K)$ :

$$
\text { for any } \bar{K} \in \mathcal{N} \quad \Lambda(\bar{K}) \leq \Lambda(K) \text {. }
$$

For any choice of descriptors $\varepsilon_{\sigma}$ an analogous $O(3)$-invariant neighborhood exists, and will be also called a wt-nbhd of $\varepsilon_{\sigma}$.

This result allows us to efficiently reduce the description of the invariance in any wt-nbhd $\mathcal{N} \subset \mathcal{Q}_{n+2}^{m}$ of the metric $K$ of an arbitrarily chosen essential $n$ lattice, and to greatly simplify the classification of its generic elastic bifurcations in $\mathcal{N}$.

For simplicity, here we consider a crystalline solid in equilibrium with a heatbath of which we only control the temperature. One can extend this treatment to 
accommodate other controls, for instance pressure, as in [24], or shear stresses, as in [18], [46], or [25]. Here an appropriate thermodynamic potential is the Helmoltz free energy of the multilattice, which is assumed to have a density per unit skeletal cell; this is a sufficiently smooth function

$$
\phi=\hat{\phi}\left(\boldsymbol{e}_{a}, \boldsymbol{p}_{r}, \theta\right)=\bar{\phi}\left(\varepsilon_{\sigma}, \theta\right),
$$

where $\theta$ denotes the absolute environmental temperature, regarded as a control. The free energy density at zero temperature coincides with the internal energy density, and it can be reasonably assumed to depend on the location of the multilattice points also at any given positive temperature. Therefore the functions $\hat{\phi}$ or $\bar{\phi}$ must have the same value on any two equivalent sets of descriptors for the same configuration, hence, for any $\mu \in \Gamma_{n+2}$, they satisfy the invariance conditions

$$
\hat{\phi}\left(m_{a}^{b} \boldsymbol{e}_{b}, \alpha_{r}^{s} \boldsymbol{p}_{s}+l_{r}^{a} \boldsymbol{e}_{a}, \theta\right)=\hat{\phi}\left(\boldsymbol{e}_{a}, \boldsymbol{p}_{r}, \theta\right), \quad \bar{\phi}\left(\mu_{\sigma}^{\tau} \varepsilon_{\tau}, \theta\right)=\bar{\phi}\left(\varepsilon_{\sigma}, \theta\right),
$$

respectively. In addition, for these functions Galilean invariance reduces to invariance under orientation-preserving isometries. In particular

$$
\hat{\phi}\left(\boldsymbol{Q} \boldsymbol{e}_{a}, \boldsymbol{Q} \boldsymbol{p}_{r}, \theta\right)=\hat{\phi}\left(\boldsymbol{e}_{a}, \boldsymbol{p}_{r}, \theta\right) \quad \text { for any } \quad \boldsymbol{Q} \in S O(3),
$$

hence

$$
\begin{aligned}
\hat{\phi}\left(\boldsymbol{e}_{a}, \boldsymbol{p}_{r}, \theta\right) & =\Phi\left(\mathrm{s}, C_{a b}, p_{r a}, \theta\right), \quad \text { for } \\
C_{a b}=\boldsymbol{e}_{a} \cdot \boldsymbol{e}_{b}, \quad p_{r a} & =\boldsymbol{p}_{r} \cdot \boldsymbol{e}_{a}, \mathrm{~s}=\operatorname{sgn}\left(\boldsymbol{e}_{1} \cdot \boldsymbol{e}_{2} \times \boldsymbol{e}_{3}\right) .
\end{aligned}
$$

For any $\mu \in \Gamma_{n+2}$ the function $\Phi$ satisfies the equality

$$
\Phi\left(\mathrm{s}, C_{a b}, p_{r a}, \theta\right)=\Phi\left(\mathrm{ss}(\mu), m_{a}^{i} C_{i j} m_{b}^{j}, m_{a}^{b}\left(\alpha_{r}^{s} p_{s b}+C_{b i} l_{r}^{i}\right), \theta\right),
$$

$m(\mu)$ denoting the $m$-component of $\mu$, and $\mathrm{s}(\mu)$ the sign of $\operatorname{det} m(\mu)$.

\section{Phase changes in a wt-nbhd}

Consider a multilattice $\mathcal{M}$ whose admissible configurations can all be described as (perhaps nonessential) $n$-lattices; a (reference) configuration in which $\mathcal{M}$ is an essential $n$-lattice, described by vectors $\varepsilon_{\sigma}^{0}=\left(\boldsymbol{e}_{a}^{0}, \boldsymbol{p}_{r}^{0}\right)$, and an $O(3)$ invariant wt-nbhd $\mathcal{N}$ of $\varepsilon_{\sigma}^{0}$, based on Proposition 1. Since no configurations of higher complexity are considered, all our results are local, and in the configuration space the nonessential multilattices form smooth submanifolds of strictly lower dimension (see [44]); and since $\mathcal{N}$ is the union of disjoint $S O(3)$-invariant neighborhoods $\mathcal{N}^{+}$and $\mathcal{N}^{-}$of $\varepsilon_{\sigma}^{0}$ and $-\varepsilon_{\sigma}^{0}$, respectively, we can assume, without loss of generality, that in $\mathcal{N}^{+}$all descriptors $\left(\boldsymbol{e}_{a}, \boldsymbol{p}_{r}\right)$ are essential, and $\mathrm{s}=\mathrm{s}^{0}:=\operatorname{sgn}\left(\boldsymbol{e}_{1}^{0} \cdot \boldsymbol{e}_{2}^{0} \times \boldsymbol{e}_{3}^{0}\right)$. The following analogues of $(13)_{1},(18)_{2}$ hold:

$$
\begin{gathered}
m_{a}^{b} \boldsymbol{e}_{b}^{0}=\boldsymbol{Q} \boldsymbol{e}_{a}^{0}, \quad \alpha_{r}^{s} \boldsymbol{p}_{s}^{0}+l_{r}^{a} \boldsymbol{e}_{a}^{0}=\boldsymbol{Q} \boldsymbol{p}_{r}^{0} \quad \text { for } \quad \boldsymbol{Q} \in P\left(\varepsilon_{\sigma}^{0}\right), \\
\hat{\phi}\left(\boldsymbol{e}_{a}^{0}, \boldsymbol{p}_{r}^{0}, \theta\right)=\hat{\phi}\left(m_{a}^{b} \boldsymbol{e}_{b}^{0}, \alpha_{r}^{s} \boldsymbol{p}_{s}^{0}+l_{r}^{a} \boldsymbol{e}_{a}^{0}, \theta\right) \quad \text { for } \quad \mu \in \Lambda\left(\varepsilon_{\sigma}^{0}\right) .
\end{gathered}
$$


Denoting by Sym the space of symmetric tensors, and by $\mathrm{Sym}^{>}$the convex cone of the positive definite ones, we normalize the orientation of the (deformed) skeletal lattices of the multilattices in $\mathcal{N}^{+}$by restricting the attention to lattice bases of the form $\boldsymbol{e}_{a}=\boldsymbol{U} \boldsymbol{e}_{a}^{0}, \boldsymbol{U} \in$ Sym $^{>}$, and define the referential shift increments $\boldsymbol{\pi}_{r}$ by the equalities

$$
\boldsymbol{p}_{r}=\boldsymbol{U}\left(\boldsymbol{p}_{r}^{0}+\boldsymbol{\pi}_{r}\right) .
$$

The following is easily proved:

$$
C_{a b}=\boldsymbol{e}_{a}^{0} \cdot \boldsymbol{C} \boldsymbol{e}_{b}^{0}, \quad \boldsymbol{C}=\boldsymbol{U}^{2}, \text { and } p_{r a}=\boldsymbol{e}_{a}^{0} \cdot \boldsymbol{C}\left(\boldsymbol{p}_{r}^{0}+\boldsymbol{\pi}_{r}\right) .
$$

Therefore in $\mathcal{N}^{+}$, where $\mathrm{s}$ is fixed,

$$
\Phi\left(\mathrm{s}, C_{a b}, p_{r a}, \theta\right)=\Psi\left(\boldsymbol{C}, \boldsymbol{\pi}_{r}, \theta\right) .
$$

From (18), (23), and (25), we have, for $\boldsymbol{Q} \in P^{+}\left(\varepsilon_{\sigma}^{0}\right), \mu \in \Lambda^{+}\left(\varepsilon_{\sigma}^{0}\right)$ :

$$
\Psi\left(\boldsymbol{C}, \boldsymbol{\pi}_{r}, \theta\right)=\Psi\left(\boldsymbol{Q}^{t} \boldsymbol{C} \boldsymbol{Q}, \alpha_{r}^{s} \boldsymbol{Q}^{t} \boldsymbol{\pi}_{s}, \theta\right), \quad \boldsymbol{Q} \varepsilon_{\sigma}^{0}=\mu_{\sigma}^{\tau} \varepsilon_{\tau}^{0} ;
$$

here $P^{+}\left(\varepsilon_{\sigma}^{0}\right)$ is the subgroup of positive-determinant elements of $P\left(\varepsilon_{\sigma}^{0}\right)$, with an analogue for the $m$ component of the elements of $\Lambda^{+}\left(\varepsilon_{\sigma}^{0}\right)$. Indeed, for any $\mu \in \Lambda^{+}\left(\varepsilon_{\sigma}^{0}\right)$,

$$
\begin{aligned}
\Psi\left(\boldsymbol{C}, \boldsymbol{\pi}_{r}, \theta\right) & =\hat{\phi}\left(\boldsymbol{e}_{a}, \boldsymbol{p}_{r}, \theta\right) \\
& =\hat{\phi}\left(m_{a}^{b} \boldsymbol{e}_{b}, \alpha_{r}^{s} \boldsymbol{p}_{s}+l_{r}^{a} \boldsymbol{e}_{a}, \theta\right) \\
& =\hat{\phi}\left(\boldsymbol{U} \boldsymbol{Q} \boldsymbol{e}_{a}^{0}, \boldsymbol{U} \boldsymbol{Q}\left(\boldsymbol{p}_{r}^{0}+\alpha_{r}^{s} \boldsymbol{Q}^{t} \boldsymbol{\pi}_{s}\right), \theta\right),
\end{aligned}
$$

from which the conclusion follows by (26) and (27).

A minor problem is that the matrix $\alpha=\left(\alpha_{r}^{s}\right)$ is not orthogonal; but it can be always expressed in terms of one such, and it is convenient to do so. For instance, denote by

$$
G:=\left\{\alpha_{i}: \mu_{i} \in \Lambda^{+}\left(\varepsilon_{\sigma}^{0}\right)\right\}
$$

the group of the submatrices $\alpha$ of the elements of $\Lambda^{+}\left(\varepsilon_{\sigma}^{0}\right)$, and by $|G|$ the order of $G$; then construct the 'metric'

$$
\begin{aligned}
g & =\frac{1}{|G|} \sum_{j=1}^{|G|} \alpha_{j}^{t} \alpha_{j}=\sum_{k=1}^{n-1} \lambda_{k}^{2} d_{k} \otimes d_{k}, \\
& =: \quad W^{2}, \quad W=\sum_{k=1}^{n-1} \lambda_{k} d_{k} \otimes d_{k},
\end{aligned}
$$

the $d_{k}$ being an orthonormal basis of eigenvectors of $g$ and $W$. It immediately follows that

$$
\alpha_{i}^{t} g \alpha_{i}=g \quad \text { for all } \quad \alpha_{i} \in G .
$$

Introduce now the group $\tilde{G}$, conjugate to $G$, consisting of the orthogonal matrices

$$
\beta_{i}=W \alpha_{i} W^{-1} \in O(n-1)
$$


and the new reference shift increments

$$
\varpi_{r}=\left(W^{-1}\right)_{r}^{s} \boldsymbol{\pi}_{s} .
$$

Then the new function $\tilde{\Psi}\left(\boldsymbol{C}, \varpi_{r}\right)=\Psi\left(\boldsymbol{C}, W_{r}^{s} \varpi_{s}\right)$ has the following invariance, when $\boldsymbol{Q}$ and $\beta$ are related to the same matrix $\mu \in \Lambda^{+}\left(\varepsilon_{\sigma}^{0}\right)$ :

$$
\tilde{\Psi}\left(\boldsymbol{C}, \varpi_{r}\right)=\tilde{\Psi}\left(\boldsymbol{Q}^{t} \boldsymbol{C} \boldsymbol{Q}, \beta_{r}^{s} \boldsymbol{Q}^{t} \varpi_{s}\right), \quad \text { for any } \quad \boldsymbol{Q} \in P^{+}\left(\varepsilon_{\sigma}^{0}\right), \beta \in \tilde{G} .
$$

At this point we apply a procedure used in [23] (see also [44]) to classify generic weak bifurcations in simple lattices: we introduce orthonormal bases $\boldsymbol{V}_{k}, k=1, \ldots, 6$, in Sym and $\boldsymbol{c}_{l}, l=1,2,3$, in $\mathbb{R}^{3}$, and the representations

$$
\boldsymbol{C}=\mathbf{1}+\sum_{k=1}^{6} y_{k} \boldsymbol{V}_{k}, \quad \varpi_{r}=\sum_{l=1}^{3} y_{r}^{l} \boldsymbol{c}_{l},
$$

so that, in particular, $\boldsymbol{C}$ is near $\mathbf{1}$ if and only if $\left(y_{1}, \ldots, y_{6}\right)$ is near $0 \in \mathbb{R}^{6}$. In the treatment of the reduced problems in $\S 4$ we will choose the basis $\left(\boldsymbol{c}_{1}, \boldsymbol{c}_{2}, \boldsymbol{c}_{3}\right)$ to coincide with $(\boldsymbol{i}, \boldsymbol{j}, \boldsymbol{k})$ introduced there, and the basis $\boldsymbol{V}_{1}, \ldots, \boldsymbol{V}_{6}$ to consist of the tensors represented in the basis $(\boldsymbol{i}, \boldsymbol{j}, \boldsymbol{k})$ by the matrices shown in (47)-(49).

By putting in a single list $\left(\mathrm{y}_{\mathrm{i}}\right)$ the $3(n+1)$ coordinates so introduced, we can define the corresponding free energy density $\hat{\Psi}\left(\mathrm{y}_{\mathrm{i}}, \theta\right)=\tilde{\Psi}\left(\boldsymbol{C}, \varpi_{r}, \theta\right)$, which then enjoys the invariance

$$
\hat{\Psi}\left(\mathrm{y}_{\mathrm{i}}, \theta\right)=\hat{\Psi}\left(\overline{\mathrm{y}}_{\mathrm{i}}, \theta\right), \quad \overline{\mathrm{y}}_{\mathrm{i}}=\mathrm{Q}_{\mathrm{ij}} \mathrm{y}_{\mathrm{j}}, \quad \mathrm{Q} \in O(3(n+1)) .
$$

By $(35)_{1}$ each matrix $Q$ is a block matrix, with a 6 by 6 and a $3(n-1)$ by $3(n-1)$ blocks, each one itself orthogonal. We denote by $\mathrm{G}$ the group of such orthogonal matrices $\mathrm{Q}$ corresponding to elements $\mu$ of $\Lambda^{+}\left(\varepsilon_{\sigma}^{0}\right)$.

In terms of $\hat{\Psi}$ the equations of equilibrium in the absence of loads are, in a convenient notation,

$$
\hat{\Psi}_{\mathrm{y}_{\mathrm{i}}}:=\frac{\partial \hat{\Psi}}{\partial \mathrm{y}_{\mathrm{i}}}\left(\mathrm{y}_{\mathrm{j}}, \theta\right)=0, \mathrm{i}=1, \ldots, 3(n+1) .
$$

We assume these conditions to hold for $\theta=\bar{\theta}$ and $\mathrm{y}_{\mathrm{i}}=0$, the latter giving the (reference) multilattice $\mathcal{M}\left(\varepsilon_{\sigma}^{0}\right)$. Consider now the second-order symmetric tensor of moduli at the transition:

$$
\mathrm{L}_{\mathrm{ij}}=\hat{\Psi}_{\mathrm{yi}_{\mathrm{i}}}(0, \bar{\theta}) ;
$$

if this is invertible, then, by the implicit function theorem, the equilibrium equations (38) have one solution $\mathrm{y}_{\mathrm{i}}=\overline{\mathrm{y}}_{\mathrm{i}}(\theta)$ for $\theta$ near $\bar{\theta}$, such that $\overline{\mathrm{y}}_{\mathrm{i}}(\bar{\theta})=0, \mathrm{i}=$ $1, \ldots, 3(n+1)$. Also, by continuity and uniqueness, all points on this equilibrium branch have the same symmetry as $\mathcal{M}\left(\varepsilon_{\sigma}^{0}\right)$.

Therefore symmetry breaking can only occur if the tensor $L$ of moduli has a nontrivial kernel. The invariance (37) forces, by differentiation, the following identity among the second derivatives of $\hat{\Psi}$ at $(0, \bar{\theta})$ :

$$
\mathrm{L}=\mathrm{Q}^{t} \mathrm{LQ} \text { for any } \mathrm{Q} \in \mathrm{G},
$$


hence the eigenspaces of $L$ are invariant under the action of G:

$$
Q^{t} L Q y=L y=\lambda y \quad \Leftrightarrow \quad L Q y=\lambda Q y .
$$

This can be interpreted by saying that invariance forces certain eigenvalues of $L$ to be equal. We restrict the attention to the case, called generic in [23] (see also [44]), in which the only conditions to be imposed on derivatives of $\hat{\Psi}$ are those guaranteeing that $(0, \bar{\theta})$ is a stable equilibrium at which bifurcation occurs, and those forced by invariance; for instance (40). In particular, the only eigenvalues of $L$ that are equal are the ones that are forced to be so by invariance; or, the eigenspaces of $L$ are irreducible invariant (i.i.) subspaces of $\mathbb{R}^{3(n+1)}$ under the action $\mathrm{y} \mapsto$ Qy of the group G, and exactly one of them is the kernel of L. Then, the condition that a stable phase exists, say, for $\theta>\bar{\theta}$ forces all the other eigenvalues to be strictly positive.

We call reduced the action of $\mathrm{G}$ on each i.i. subspace, and also call reduced the group representing such action on that subspace. If one chooses the basis above aligned with a choice of i.i. subspaces, then each matrix $Q$ is a block matrix, each orthogonal block corresponding to an i.i. subspace, and representing an element of the reduced group on that subspace.

The fact that the action of $\mathrm{G}$ does not mix the first 6 and the last $3(n-1)$ coordinates (see (35)), implies that the i.i. subspaces of $\mathbb{R}^{3(n+1)}$ are necessarily of either one of the forms

$$
\mathcal{V}_{1} \times\{0\} \quad \text { or } \quad\{0\} \times \mathcal{V}_{2},
$$

where $\mathcal{V}_{1}\left[\mathcal{V}_{2}\right]$ is an i.i. subspace of $\mathbb{R}^{6}\left[\right.$ of $\left.\mathbb{R}^{3(n-1)}\right]$ and $0 \in \mathbb{R}^{3(n-1)}\left[0 \in \mathbb{R}^{6}\right]$. Case $(42)_{1}$ corresponds to configurational transitions, in which the motif follows the deformation of the skeleton, at least in the beginning. Case $(42)_{2}$ describes structural transitions, which are driven by the deformation of the motif, followed by a suitable consequent deformation of the skeleton. We now follow a classical procedure: we determine the i.i. subspaces of $\mathbb{R}^{6}\left[\right.$ of $\left.\mathbb{R}^{3(n-1)}\right]$ for case $(42)_{1}$ $\left[(42)_{2}\right]$, and then consider the corresponding reduced problem; a description of these can be found in [30], [23], [47], [44].

\section{The case of $\beta$-quartz}

At low pressures quartz exhibits two stable phases, called 'low' (or trigonal, or $\alpha$-) quartz and 'high' (or hexagonal, or $\beta$-) quartz; at room pressure, these phases are observed below and above about $574^{\circ} \mathrm{C}$, respectively.

We follow [36] (and [44]) by assuming that in any configuration of the $\mathrm{SiO}_{2}$ structure the positions of the $\mathrm{Si}$ atoms be compatible with the definition of a 3-lattice, and neglect the oxigens; thus we describe the crystalline structure of both quartz phases by a monatomic 3-lattice, whose points are the positions of the $\mathrm{Si}$ atoms in the $\mathrm{SiO}_{2}$ lattice. In the literature the $\alpha-\beta$ transition is attributed to a suitable deformation of the tetrahedra having the center at a Si atom, and the four nearest $\mathrm{O}$ atoms as vertices. Here this deformation is only described by the displacement of the $\mathrm{Si}$ atoms. 


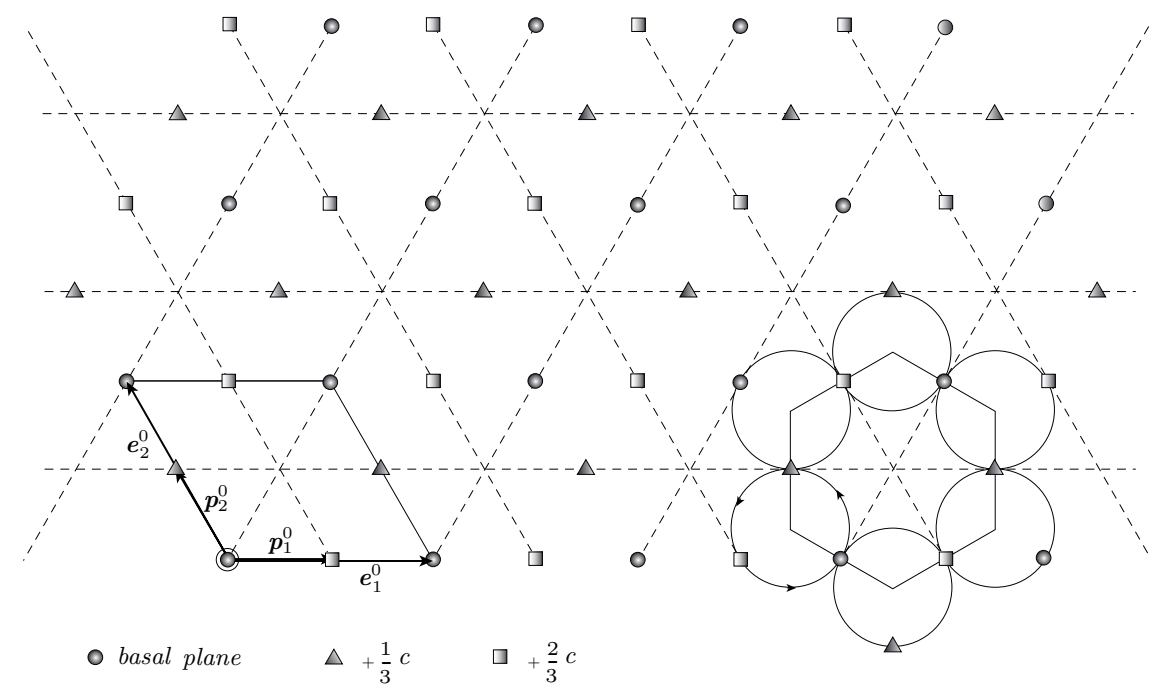

Figure 4: Projection onto the basal plane of the $\mathrm{Si}$ atoms in right-handed $\beta$ quartz, and of the descriptors $\varepsilon_{\sigma}^{0}=\left(\boldsymbol{e}_{a}^{0}, \boldsymbol{p}_{1}^{0}, \boldsymbol{p}_{2}^{0}\right)$ for the 3-lattice given by (43) and (45) with $\lambda>0$

In both $\alpha$ - and $\beta$-quartz the skeletal lattice type is hexagonal. A common choice of lattice vectors is the following:

$$
\boldsymbol{e}_{1}=(a, 0,0), \quad \boldsymbol{e}_{2}=\left(-\frac{a}{2}, \frac{\sqrt{3} a}{2}, 0\right), \quad \boldsymbol{e}_{3}=(0,0, c),
$$

in an orthonormal basis $(\boldsymbol{i}, \boldsymbol{j}, \boldsymbol{k})$. The rotational subgroup of the corresponding hexagonal holohedry is

$$
\mathcal{H}_{k}=\left\{1, \boldsymbol{R}_{i}^{\pi}, \boldsymbol{R}_{j}^{\pi}, \boldsymbol{R}_{k}^{\pi}, \boldsymbol{R}_{k}^{\frac{\pi}{3}}, \boldsymbol{R}_{k}^{\frac{2 \pi}{3}}, \boldsymbol{R}_{k}^{\frac{4 \pi}{3}}, \boldsymbol{R}_{k}^{\frac{5 \pi}{3}}, \boldsymbol{R}_{i \pm \sqrt{3} j}^{\pi}, \boldsymbol{R}_{\sqrt{3} i \pm j}^{\pi}\right\}
$$

where $\boldsymbol{R}_{v}^{\omega}$ denotes the rotation by the angle $\omega$ about the direction of the vector $\boldsymbol{v}$. In the crystallographic literature the plane of $\boldsymbol{e}_{1}$ and $\boldsymbol{e}_{2}$ is called the basal plane, and the direction of $\boldsymbol{e}_{3}$ (and of $\boldsymbol{k}$ ) is called the (hexagonal) optic axis.

One of the two possible (enantiomorphic) 3-lattice structures of $\beta$-quartz at the transition temperature $\theta_{0}$ has descriptors $\varepsilon_{\sigma}^{0}, \sigma=1, \ldots, 5$, where the lattice vectors $\boldsymbol{e}_{a}^{0}$ are given by (43) for suitable choices $a_{0}, c_{0}$, of $a$ and $c$, and the shifts are

$$
p_{1}^{0}=\frac{1}{2} e_{1}^{0}+\frac{2}{3} e_{3}^{0}, \quad p_{2}^{0}=\frac{1}{2} e_{2}^{0}+\frac{1}{3} e_{3}^{0} .
$$

Fig. 4 shows the projection of the 3-lattice $\mathcal{M}\left(\varepsilon_{\sigma}^{0}\right)$ onto the basal plane of $\boldsymbol{e}_{1}^{0}$ and $\boldsymbol{e}_{2}^{0}$ orthogonal to the optic axis $\boldsymbol{e}_{3}^{0}$.

James in [36] (see also [44]) constructs the above 3-lattice structure of $\beta$ quartz as follows: consider a regular hexagonal planar honeycomb of edge $d_{0}=$ 
$\frac{\sqrt{3}}{3} a_{0}$, one cell of which is drawn by a plain line in the lower right part of Fig. 4. For each one of its vertices consider a right-handed (with respect to $\boldsymbol{k}$ ) circular helix of radius $\frac{d_{0}}{2}$ and pitch $c_{0}$, whose axis goes through that vertex and is orthogonal to the plane of the honeycomb. It is possible to arrange the right-handed helices in such a way that each one meets the neighboring three in equally spaced points along the helix itself, each point having the same projection onto the plane of the honeycomb as the third point following it. Each intersection point of the helices is the site of a $\mathrm{Si}$ atom. The circles in Fig. 4 are the projections of the helices in the plane of the honeycomb, which is the basal plane generated by $\boldsymbol{e}_{1}^{0}, \boldsymbol{e}_{2}^{0}$.

The crystal class $P\left(\varepsilon_{\sigma}^{0}\right)<S O(3)$ of this 3-lattice is the rotational subgroup $\mathcal{H}_{k}$ (see (44)) of the hexagonal holohedry $P\left(\boldsymbol{e}_{a}^{0}\right)$, and is generated for instance by $\boldsymbol{R}_{k}^{\pi / 3}$ and $\boldsymbol{R}_{i}^{\pi}$. This class is called hexagonal trapezohedral, is denoted by 622 in [35], and is the actual crystal class of $\beta$-quartz, so that the monatomic 3-lattice $\mathcal{M}\left(\varepsilon_{\sigma}^{0}\right)$ gives a good approximation of the actual structure of this quartz phase.

Case $(42)_{1}$

We follow [23], in the notation of [44] to which we address for more details.

(1) The set $\hat{C}\left(\boldsymbol{e}_{a}^{0}\right)$ of symmetric tensors left fixed by the transformation $\boldsymbol{E} \mapsto$ $\boldsymbol{Q}^{t} \boldsymbol{E} \boldsymbol{Q}$ of $S y m$ form the 2-dimensional subspace of Sym represented in the basis $(\boldsymbol{i}, \boldsymbol{j}, \boldsymbol{k})$ by matrices of the form

$$
\left(\begin{array}{lll}
\alpha & 0 & 0 \\
0 & \alpha & 0 \\
0 & 0 & \beta
\end{array}\right)
$$

a basis for $\hat{C}\left(\boldsymbol{e}_{a}^{0}\right)$ is given, for instance, by the orthonormal vectors

$$
\boldsymbol{V}_{1}=\frac{1}{\sqrt{2}}\left(\begin{array}{ccc}
1 & 0 & 0 \\
0 & 1 & 0 \\
0 & 0 & 0
\end{array}\right) \quad \text { and } \quad \boldsymbol{V}_{2}=\left(\begin{array}{ccc}
0 & 0 & 0 \\
0 & 0 & 0 \\
0 & 0 & 1
\end{array}\right)
$$

The i.i. subspaces contained in $\hat{C}\left(\boldsymbol{e}_{a}\right)$ are those and only those that are 1dimensional. In each one of them the reduced action is trivial, the bifurcation point is a turning (or limit) point, with change of stability but not of symmetry.

(2) In the orthogonal complement $\hat{C}\left(\boldsymbol{e}_{a}^{0}\right)^{\perp}$ of $\hat{C}\left(\boldsymbol{e}_{a}^{0}\right)$ there are two mutually orthogonal 2-dimensional i.i. subspaces $\mathcal{V}_{1}, \mathcal{V}_{2}$, generated by $\boldsymbol{V}_{3}, \boldsymbol{V}_{4}$ and $\boldsymbol{V}_{5}, \boldsymbol{V}_{6}$, respectively, with

$$
\begin{array}{ll}
\boldsymbol{V}_{3}=\frac{1}{\sqrt{2}}\left(\begin{array}{lll}
0 & 1 & 0 \\
1 & 0 & 0 \\
0 & 0 & 0
\end{array}\right), \quad \boldsymbol{V}_{4}=\frac{1}{\sqrt{2}}\left(\begin{array}{ccc}
1 & 0 & 0 \\
0 & -1 & 0 \\
0 & 0 & 0
\end{array}\right), \\
\boldsymbol{V}_{5}=\frac{1}{\sqrt{2}}\left(\begin{array}{lll}
0 & 0 & 1 \\
0 & 0 & 0 \\
1 & 0 & 0
\end{array}\right), \quad \boldsymbol{V}_{6}=\frac{1}{\sqrt{2}}\left(\begin{array}{lcc}
0 & 0 & 0 \\
0 & 0 & 1 \\
0 & 1 & 0
\end{array}\right) .
\end{array}
$$


(3) The reduced group $\mathcal{P}_{1}$ on $\mathcal{V}_{1}$ is the symmetry group of an equilateral triangle in $\mathbb{R}^{2}$ with center at the origin and a vertex on the second coordinate axis; it has order six and is generated $b^{2}$

$$
f:=\left(\begin{array}{cc}
-1 & 0 \\
0 & 1
\end{array}\right) \approx \boldsymbol{R}_{i}^{\pi} \text { and } r(2 \pi / 3) \approx \boldsymbol{R}_{k}^{\frac{\pi}{3}}, \quad r(\omega):=\left(\begin{array}{cc}
\cos \omega & -\sin \omega \\
\sin \omega & \cos \omega
\end{array}\right) .
$$

Thus the action of $\mathcal{H}_{k}$ on the typical element of $\mathcal{V}_{1}$ produces six monoclinic variants, the symmetry axis being $\boldsymbol{k}$. In addition, $\mathcal{V}_{1}$ contains three 1-dimensional base-centered orthorhombic subspaces, whose crystal class is rhombic disphenoidal (222 in [35]).

The reduced bifurcation diagram consists in three transcritical bifurcating curves which belong to the aforementioned subspaces and are all unstable. This is detailed in [44], where it is also shown how these curves can be re-stabilized, thus producing a subcritical bifurcation.

(4) The reduced group $\mathcal{P}_{2}$ on $\mathcal{V}_{2}$ is the group of symmetries of a regular hexagon in $\mathbb{R}^{2}$ with center at the origin and a vertex on the first axis, has order twelve and is generated by

$$
f \approx \boldsymbol{R}_{i}^{\pi} \text { and } \quad r(\pi / 3) \approx \boldsymbol{R}_{k}^{\frac{5 \pi}{3}}
$$

Thus the action of the hexagonal holohedry on the typical element of $\mathcal{V}_{2}$ produces twelve triclinic variants.

In $\mathcal{V}_{2}$ there are two triples of symmetry-related 1-dimensional subspaces made of centered monoclinics, whose crystal class is monoclinic sphenoidal (2 in [35]). The bifurcating curves consist in two triples of pitchforks, each one in one of these triples of monoclinic subspaces. For a triple of pitchforks to be stable it is necessary that it be supercritical: it must exist for $\theta \leq \theta_{0}$ under the assumed stability of the high-symmetry phase for $\theta>\theta_{0}$; then necessarily also the other triple is supercritical, and exactly one of them is stable.

Case $(42)_{2}$

We denote by $\alpha_{k}^{\pi / 3}$ the submatrix $\alpha$ of the matrix $\mu_{k}^{\pi / 3} \in \Lambda^{+}\left(\varepsilon_{\sigma}^{0}\right)$ that corresponds to the rotation $\boldsymbol{R}_{k}^{\pi / 3}$ according to (13), etc., $\alpha_{\mathbf{1}}$ denoting the 2 by 2 identity. Using the expressions of the generators $\mu_{k}^{\pi / 3}$ and $\mu_{i}^{\pi}$ reported in [44], we have

$$
\begin{gathered}
\alpha_{1}=\left(\begin{array}{ll}
1 & 0 \\
0 & 1
\end{array}\right)=\alpha_{k}^{\pi}, \quad \alpha_{k}^{\pi / 3}=\left(\begin{array}{cc}
-1 & -1 \\
1 & 0
\end{array}\right)=\alpha_{k}^{4 \pi / 3}, \\
\alpha_{k}^{2 \pi / 3}=\left(\begin{array}{rr}
0 & 1 \\
-1 & -1
\end{array}\right)=\alpha_{k}^{5 \pi / 3}, \quad \alpha_{i}^{\pi}=\left(\begin{array}{cc}
-1 & -1 \\
0 & 1
\end{array}\right)=\alpha_{j}^{\pi}, \\
\alpha_{\sqrt{3} i-j}^{\pi}=\left(\begin{array}{ll}
0 & 1 \\
1 & 0
\end{array}\right)=\alpha_{i+\sqrt{3} j}^{\pi}, \quad \alpha_{i-\sqrt{3} j}^{\pi}=\left(\begin{array}{rr}
1 & 0 \\
-1 & -1
\end{array}\right)=\alpha_{\sqrt{3} i+j}^{\pi} .
\end{gathered}
$$

\footnotetext{
${ }^{2}$ Here and below $\approx$ means 'represented by' or 'representing'.
} 
Therefore, by (31),

$$
g=\left(\begin{array}{cc}
\frac{4}{3} & \frac{2}{3} \\
\frac{2}{3} & \frac{4}{3}
\end{array}\right) \text { and } W=\frac{1}{3 \sqrt{2}}\left(\begin{array}{cc}
3+\sqrt{3} & 3-\sqrt{3} \\
3-\sqrt{3} & 3+\sqrt{3}
\end{array}\right),
$$

from which, by (33) and in obvious notation,

$$
\begin{gathered}
\beta_{1}=\left(\begin{array}{ll}
1 & 0 \\
0 & 1
\end{array}\right)=\beta_{k}^{\pi}, \quad \beta_{k}^{\pi / 3}=-\frac{1}{2}\left(\begin{array}{cc}
1 & \sqrt{3} \\
-\sqrt{3} & 1
\end{array}\right)=\beta_{k}^{4 \pi / 3}, \\
\beta_{k}^{2 \pi / 3}=-\frac{1}{2}\left(\begin{array}{cc}
1 & -\sqrt{3} \\
\sqrt{3} & 1
\end{array}\right)=\beta_{k}^{5 \pi / 3}, \quad \beta_{i}^{\pi}=-\frac{1}{2}\left(\begin{array}{cc}
\sqrt{3} & 1 \\
1 & -\sqrt{3}
\end{array}\right)=\beta_{j}^{\pi}, \\
\beta_{\sqrt{3} i-j}^{\pi}=\left(\begin{array}{ll}
0 & 1 \\
1 & 0
\end{array}\right)=\beta_{i+\sqrt{3} j}^{\pi}, \quad \beta_{i-\sqrt{3} j}^{\pi}=-\frac{1}{2}\left(\begin{array}{cc}
-\sqrt{3} & 1 \\
1 & \sqrt{3}
\end{array}\right)=\beta_{\sqrt{3} i+j}^{\pi} .
\end{gathered}
$$

Consider now the action induced by $(37)_{2}$ on the 6 -dimensional space of shift components, with typical element $\left(a_{1}, a_{2}, a_{3}, b_{1}, b_{2}, b_{3}\right)$ representing the pair $\left(\varpi_{1}, \varpi_{2}\right)$. One can check that this action transforms into themselves the subspaces $\mathcal{W}_{1}$ and $\mathcal{W}$ of the form $\left(0,0, a_{3}, 0,0, b_{3}\right)$ and $\left(a_{1}, a_{2}, 0, b_{1}, b_{2}, 0\right)$, respectively.

The 2-dimensional subspace $\mathcal{W}_{1}$, described by the pair $\left(a_{3}, b_{3}\right)$, is irreducible invariant, and consists of monoclinic 3-lattices, with axis $\boldsymbol{k}$. The reduced group on $\mathcal{W}_{1}$ has order 6 , is generated by the matrices

$$
\frac{1}{2}\left(\begin{array}{cc}
\sqrt{3} & 1 \\
1 & -\sqrt{3}
\end{array}\right) \approx \boldsymbol{R}_{i}^{\pi} \approx \boldsymbol{R}_{j}^{\pi} \quad \text { and } \quad r(4 \pi / 3) \approx \boldsymbol{R}_{k}^{\frac{\pi}{3}},
$$

and is the symmetry group of an equilateral triangle in $\mathbb{R}^{2}$ centered at the origin and having a vertex on the bisectrix of the second and fourth quadrant. Therefore, to within a rotation by $\pi / 4$ of the coordinates, this reduced problem is the same as the one in item (3) of Case (42) $)_{1}$. As there, the bifurcation diagram consists of three unstable transcritical bifurcating curves of orthorhombic 222 symmetry. For instance, the orthorhombic axes (besides $\boldsymbol{k}$ ) are $\boldsymbol{i}$ and $\boldsymbol{j}$ for the choice of shifts

$$
\boldsymbol{\pi}_{1}=2 \lambda \boldsymbol{k}=2 \boldsymbol{\pi}_{2}, \quad \lambda \in \mathbb{R} .
$$

This can be obtained from the corresponding 1-dimensional subspace of $\mathcal{W}_{1}$, which has the form

$$
\left(a_{3}, b_{3}\right)=\gamma(2+\sqrt{3}, 1), \quad \gamma \in \mathbb{R},
$$

by (34) and (55), or directly from the condition - see (28)

$$
\boldsymbol{R}_{i}^{\pi} \boldsymbol{\pi}_{r}=\left(\alpha_{i}^{\pi}\right)_{r}^{s} \boldsymbol{\pi}_{s}
$$

or, equivalently, from its analogue for $\boldsymbol{R}_{j}^{\pi}$.

The subspace $\mathcal{W}$ decomposes into the orthogonal sum of three i.i. subspaces, $\mathcal{W}_{2}, \mathcal{W}_{3}, \mathcal{W}_{4}$, the first two of dimension 1 , the third of dimension 2. They are respectively generated by

$$
w_{2}=(-1,2+\sqrt{3}, 0,2+\sqrt{3}, 1,0),
$$




$$
\begin{gathered}
w_{3}=(2+\sqrt{3}, 1,0,1,-2-\sqrt{3}, 0), \quad \text { and } \\
w_{4}=(1,0,0,0,1,0), w_{4}^{\prime}=(0,1,0,-1,0,0) .
\end{gathered}
$$

The reduced group on $\mathcal{W}_{2}\left[\mathcal{W}_{3}\right]$ is $\{1,-1\}$; for instance

$$
1 \approx \boldsymbol{R}_{i}^{\pi}\left[1 \approx \boldsymbol{R}_{j}^{\pi}\right] \text { and } \quad-1 \approx \boldsymbol{R}_{k}^{\pi / 3} \approx \boldsymbol{R}_{k}^{\pi}
$$

Therefore, as is known, the bifurcation diagram is the standard pitchfork. A fourth-order polynomial energy is sufficient to capture the qualitative features of a (supercritical) second-order bifurcation, while a subcritical first-order one, as in the case of quartz, requires a sixth-order polynomial (see for instance [23], [24] or [44]). In $\mathcal{W}_{2}$ [in $\mathcal{W}_{3}$ ] the crystal class of the bifurcating multilattices is trigonal trapezohedral (32 in [35]), with $\boldsymbol{k}$ as 3-fold axis; the additional generator of the point group is $\boldsymbol{R}_{i}^{\pi}$ [is $\boldsymbol{R}_{j}^{\pi}$ ].

The reduced group on $\mathcal{W}_{4}$ has order 12 , is generated by the matrices

$$
\frac{1}{2}\left(\begin{array}{cc}
\sqrt{3} & 1 \\
1 & -\sqrt{3}
\end{array}\right) \approx \boldsymbol{R}_{i}^{\pi} \text { and } r(\pi / 3) \approx \boldsymbol{R}_{k}^{\frac{\pi}{3}},
$$

and is the symmetry group of a regular hexagon in $\mathbb{R}^{2}$ which is centered at the origin and has a vertex on the bisectrix of the second and fourth quadrant (compare with (59)). Therefore, to within a rotation by $\pi / 4$ of the coordinates, this reduced problem is the same as the one in item (4) of Case (42) 1 and has the same qualitative bifurcation diagram. All the bifurcating branches have monoclinic 2 symmetry.

We now analyze in detail the two trigonal trapezohedral subspaces $\mathcal{W}_{2}$ and $\mathcal{W}_{3}$. Using (34) and (55), the reference shift increments corresponding to $\mathcal{W}_{2}$ are, in terms of real parameters $\bar{\lambda}, \lambda^{\prime}$,

$$
\begin{gathered}
\boldsymbol{\pi}_{1}=\bar{\lambda}(0,2(3+\sqrt{3}) / 3,0)=2(1+\sqrt{3}) \lambda^{\prime}\left(\boldsymbol{e}_{1}^{0}+2 \boldsymbol{e}_{2}^{0}\right) / 3, \\
\boldsymbol{\pi}_{2}=\bar{\lambda}(1+\sqrt{3},(3+\sqrt{3}) / 3,0)=2(1+\sqrt{3}) \lambda^{\prime}\left(2 \boldsymbol{e}_{1}^{0}+\boldsymbol{e}_{2}^{0}\right) / 3 .
\end{gathered}
$$

Equivalently, by (25), denoting by $\boldsymbol{p}_{r}^{+}$the present shifts, with $\boldsymbol{p}_{r}=\boldsymbol{U} \boldsymbol{p}_{r}^{0}, \boldsymbol{e}_{a}=$ $\boldsymbol{U} \boldsymbol{e}_{a}^{0}$, and $\lambda$ a real parameter,

$$
\boldsymbol{p}_{1}^{+}=\boldsymbol{p}_{1}+\lambda\left(\boldsymbol{e}_{1}+2 \boldsymbol{e}_{2}\right), \quad \boldsymbol{p}_{2}^{+}=\boldsymbol{p}_{2}+\lambda\left(2 \boldsymbol{e}_{1}+\boldsymbol{e}_{2}\right)
$$

These shifts represent deformed $\beta$-quartz for $\lambda=0$, while for $\lambda \neq 0$ they give the $\mathcal{M}\left(\varepsilon_{\sigma}^{+}\right)$3-lattice model for trigonal trapezohedral $\alpha$-quartz proposed in [36], and used also in [24] and [44]. The projection of this 3-lattice onto the (basal) plane of $\boldsymbol{e}_{1}$ and $\boldsymbol{e}_{2}$ is sketched in Fig. 5 for $\lambda>0$.

Based on [36], we recall how the $\alpha$-quartz structure can be obtained by deforming the helices described above for $\beta$-quartz. If the reference $\beta$-quartz helices, of radius $d_{0} / 2$, maintain their axes while being radially stretched, so that their radius becomes larger than $d_{0} / 2$, then any point of initial intersection of two neighboring helices splits into two alternative intersections. For the actual 


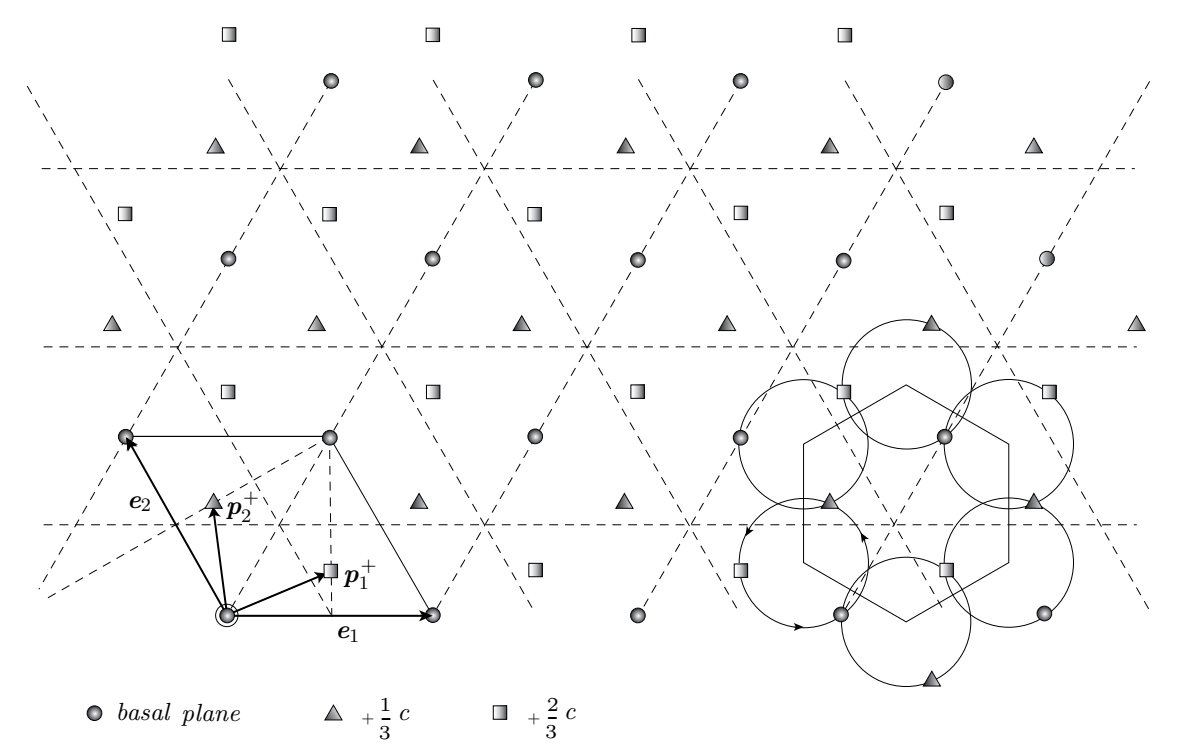

Figure 5: Projection as in Fig. 4 for the right-handed $\alpha$-quartz structure, and of the descriptors $\boldsymbol{\varepsilon}_{\sigma}^{+}=\left(\boldsymbol{e}_{a}, \boldsymbol{p}_{1}^{+}, \boldsymbol{p}_{2}^{+}\right)$for the 3-lattice given by (43) and (70) with $\boldsymbol{U}=\mathbf{1}$ and $\lambda>0$

$\alpha$-quartz structure, which is uniformly stretched by some $\boldsymbol{U} \in$ Sym $^{>}$whose representative matrix in the basis $(\boldsymbol{i}, \boldsymbol{j}, \boldsymbol{k})$ has the form (46), the projections of these intersections onto the basal plane are shown in the lower right part of Fig. 5. To maintain the threefold rotational symmetry about the normal to the honeycomb, the $\mathrm{Si}$ atoms must still be evenly spaced on the helices on such intersections, each atom being on the same vertical (with respect to the basal plane) as the third atom following it on the helix. There are exactly two ways in which this can happen: on an arbitrarily selected helix Si atoms are placed on either the first or the second of its possible intersections with one of the neighboring helices; either choice forces in a unique way the atoms on the other neighboring helices to be all placed on the second or on the first of their intersections, etc., up to completion of the whole structure. Fig. 5 shows one of the configurations obtained in this way, namely $\mathcal{M}\left(\varepsilon_{\sigma}^{+}\right)$with shifts given by (70) for $\lambda>0$. For fixed $\lambda$, the other possibility is given by the Dauphiné twin $\mathcal{M}\left(\varepsilon_{\sigma}^{-}\right)$, where the $\varepsilon_{\sigma}^{-}=\left(\boldsymbol{e}_{a}, \boldsymbol{p}_{1}^{-}, \boldsymbol{p}_{2}^{-}\right)$have the same lattice vectors $\boldsymbol{e}_{a}$ as $\varepsilon_{\sigma}^{+}$, and shifts

$$
\boldsymbol{p}_{1}^{-}=\boldsymbol{p}_{1}-\lambda\left(\boldsymbol{e}_{1}+2 \boldsymbol{e}_{2}\right), \quad \boldsymbol{p}_{2}^{-}=\boldsymbol{p}_{2}-\lambda\left(2 \boldsymbol{e}_{1}+\boldsymbol{e}_{2}\right),
$$

with the same $\lambda$ as in (70). These two configurations correspond to symmetryrelated points on the bifurcated branches of the pitchfork in the $\mathcal{W}_{2}$ subspace mentioned above. We address the reader to [44] for mode details on Dauphiné twins, and only recall that the twin multilattice $\mathcal{M}\left(\varepsilon_{\sigma}^{-}\right)$can be obtained from 
$\mathcal{M}\left(\varepsilon_{\sigma}^{+}\right)$by means of the rotation $\boldsymbol{R}_{k}^{\pi}$, of order 2 (see also (66)).

Using (34) and (55), the reference shift increments corresponding to elements of the subspace $\mathcal{W}_{3}$ are, in terms of real parameters $\bar{\mu}, \mu^{\prime}$,

$$
\begin{gathered}
\boldsymbol{\pi}_{1}^{\prime}=4 \bar{\mu}(3+\sqrt{3}, 0,0)=4 \mu^{\prime}(3+\sqrt{3}) \boldsymbol{e}_{1}^{0}, \\
\boldsymbol{\pi}_{2}^{\prime}=4 \bar{\mu}(3+\sqrt{3})(1 / 2,-\sqrt{3} / 2,0)=-4 \mu^{\prime}(3+\sqrt{3}) \boldsymbol{e}_{2}^{0} .
\end{gathered}
$$

Equivalently, by (25), denoting by $\boldsymbol{p}_{r}^{\prime}$ the corresponding present shifts,

$$
\boldsymbol{p}_{1}^{\prime}=\boldsymbol{p}_{1}+\mu \boldsymbol{e}_{1}, \quad \boldsymbol{p}_{2}^{\prime}=\boldsymbol{p}_{2}-\mu \boldsymbol{e}_{2},
$$

with $\mu$ a real parameter. We have deformed $\beta$-quartz for $\mu=0$, while symmetryrelated points on the bifurcated branches of the pitchfork in this subspace correspond to opposite values of $\mu \neq 0$ in the shifts given by (74). The related multilattices, say $\mathcal{M}\left(\varepsilon_{\sigma}^{\prime}\right)$ and $\mathcal{M}\left(\varepsilon_{\sigma}^{\prime \prime}\right)$, are another example of shuffle twins, very similar to the Dauphiné twins described above. In particular, the twin multilattice $\mathcal{M}\left(\varepsilon_{\sigma}^{\prime \prime}\right)$ can be obtained from $\mathcal{M}\left(\varepsilon_{\sigma}^{\prime}\right)$ by means of the same rotation $\boldsymbol{R}_{k}^{\pi}$, of order 2, which relates the Dauphiné twins (see (66)).

Also in this case one can describe the low-symmetry phase and the twins in terms of deformation of the reference $\beta$-quartz helices. Now the radius of those helices is shrinked, becoming less than $d_{0} / 2$, hence neighboring helices do not intersect anymore. Fig. 6 shows one of the possible arrangements of the actual helices; looking at the hexagon drawn in the lower right corner, the other possibility - which gives the twinned configuration - is obtained by exchanging the occupied and the nonoccupied helices in that hexagon, and consequently in the whole structure.

In his (2001) paper, among other things, Ericksen tackles the problem of finding an alternative to the so-called incommensurate phase, which is introduced in the physical literature to explain certain features of the $\alpha-\beta$ transition in quartz. He looks for configurations described by a 3-lattice with hexagonal skeleton and shifts such that the symmetry of the structure consists in the threefold axis $\boldsymbol{k}$ alone. The reference shift increments must have the form

$$
\boldsymbol{\pi}_{1}=(\lambda+\mu) \boldsymbol{e}_{1}^{0}+2 \lambda \boldsymbol{e}_{2}^{0}, \quad \boldsymbol{\pi}_{2}=2 \lambda \boldsymbol{e}_{1}^{0}+(\lambda-\mu) \boldsymbol{e}_{2}^{0},
$$

for $\lambda$ and $\mu$ varying arbitrarily over the reals; this corresponds in fact to the 2-dimensional orthogonal sum $\mathcal{W}_{2} \boxplus \mathcal{W}_{3}$ of the above i.i. subspaces $\mathcal{W}_{2}$ and $\mathcal{W}_{3}$. In that 2-dimensional space Ericksen, generalizing an example in [47], introduces a sextic polynomial (reduced) potential in the variables $\lambda, \mu$, with the coefficients of $\lambda^{2}$ and $\mu^{2}$ depending affinely on environmental pressure $p$ and temperature $\theta$, and all the other coefficients constant. The related bifurcation analysis shows the existence of four phases, labelled I ( $\lambda=0=\mu, \beta$-phase), II $\left(\lambda \neq 0=\mu, \alpha\right.$-phase, our subspace $\left.\mathcal{W}_{2}\right)$, III $\left(\lambda=0 \neq \mu\right.$, our subspace $\left.\mathcal{W}_{3}\right)$, and IV $\left(\lambda \neq 0 \neq \mu\right.$, the complement of the previous phases in $\left.\mathcal{W}_{2} \boxplus \mathcal{W}_{3}\right)$. According to Ericksen, phases II and III and their twins are rather similar, so that one could be confused for the other. For instance, the twins can be described for both phases in terms of the same twinning operation $\boldsymbol{R}_{k}^{\pi}$. 


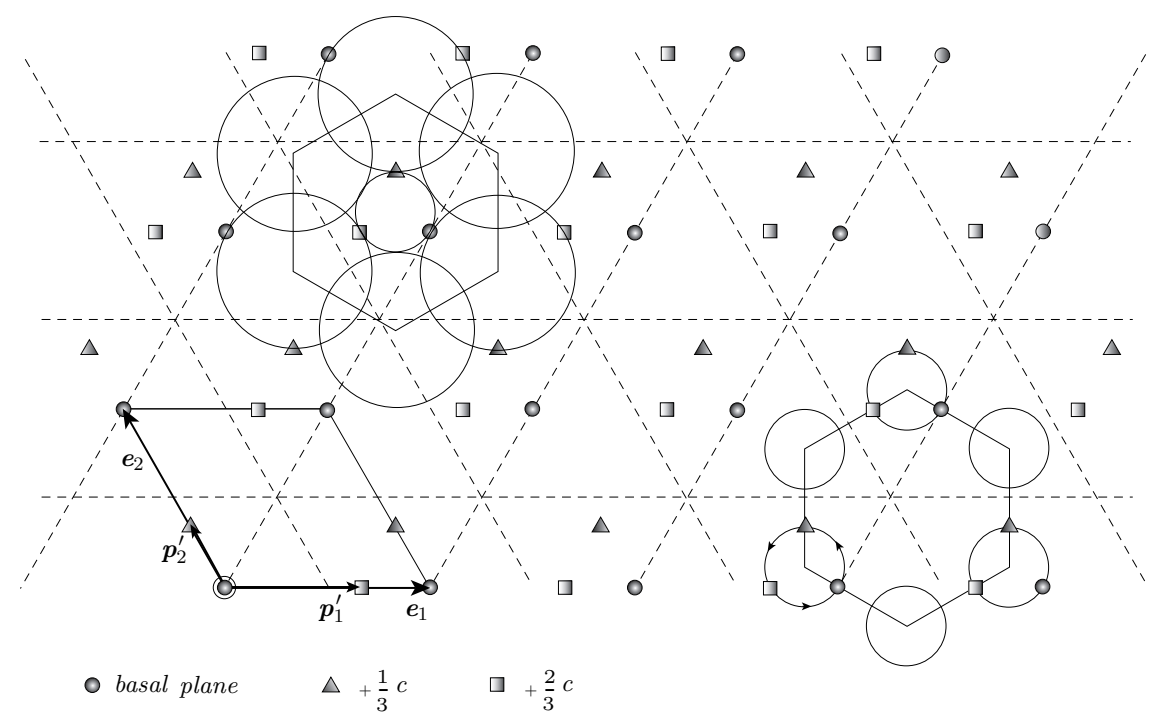

Figure 6: Projection as in Fig. 5 for the right-handed quartz structure with shifts given by (74) for $\boldsymbol{U}=\mathbf{1}$ and $\mu>0$

Ericksen uses the sextic potential to describe the transition from $\beta$ - to $\alpha$ quartz, at constant pressure when temperature is lowered, by means of a secondorder transition from phase I to phase III, followed by a first-order transition between phases III and II.

Notice that a direct transition from I to IV corresponds to bifurcating into the orthogonal sum of $\mathcal{W}_{2}$ and $\mathcal{W}_{3}$ in an (initial) direction contained in neither $\mathcal{W}_{2}$ nor $\mathcal{W}_{3}$, which requires the vanishing of both the eigenvalues of $\mathrm{L}$ corresponding to those eigenspaces. This is not forced by symmetry, hence cannot generically occur with one control parameter, as the kernel of $L$ is a reducible invariant subspace of $\mathbb{R}^{6}$.

We address the reader to [24] for details, in particular for a comparison with certain formulae of [47] involving bifurcation into a reducible invariant subspace, of interest in his bifurcation analysis, and for comments on the incommensurate phase.

\section{$5 \quad$ A personal tribute to Clifford Truesdell}

The fact that this paper appears in a volume dedicated to the memory of Clifford Truesdell gives me the opportunity to acknowledge, in addition to my debt of gratitude and admiration toward him, some of his merits that are not well known as well as very unusual. Additional evidence of these merits was recently given by Bressan in [17], who mentioned the existence of various unpublished 
results obtained within a research program strongly supported by Truesdell. Much earlier, Truesdell sent me a confidential copy of his 1986 letter to the Accademia dei Lincei, in which he expresses his support to that program. ${ }^{3}$ An outline of the content of this letter will be given in item (D) below.

Among his various merits, Truesdell was very interested in some research fields different from those in which he himself or his associates were active. For the purpose of this tribute, he was interested in the logic of modality, that is, of possibility and hence of necessity, especially in its version ${ }^{4}$ introduced by Bressan in [5] under the name of physical possibility to write rigorous foundations of classical particle mechanics based on ideas of Mach and Painlevé ([40]). Truesdell supported the extension of this research to continuous media, as shown by his 1986 letter to the Accademia, and was able to base some polemic considerations in the same field on very strong arguments (Part V, Philosophy, essay 39: Suppesian stews (1980/1981), of [48]). These assertions are supported by items (A) to (E) below.

(A) Truesdell accepted as appendix G6 of his well known book [49] my paper [42], in which I explicitly refer to Bressan's theory of modal logic, presented in progressively refined versions in [5], [6], [8]. It is true that I use these versions from an intuitive point of view, ${ }^{5}$ but Bressan's physical possibility is a key ingredient in definitions and axioms.

In his conference paper [17] Bressan emphasized how the use of modalities was considered highly exceptional, ${ }^{6}$ as exemplified by the important interdisciplinary (in the sense of footnote 2) 1959 meeting in Paris, which was supported by the International Society of History and Philosophy of Science, and whose proceedings were edited by the mathematical physicist Destouches ([22]). More comments will be given in (E) below.

(B) The value of Bressan's theory of modal logic is apparent, in particular, in the proceedings of the 1972 PSA meeting in Lansing - see [7]. ${ }^{7}$ The

\footnotetext{
'Truesdell was 'Socio Straniero' of the Academy, and had been asked for his opinion about the publication of [12], where the program of extension mentioned in the next paragraph is started.

${ }^{4}$ The mentioned version of possibility: (1) has some intuitive features by means of which it is regarded as a primitive concept; (2) it makes axiomatization of physics rigorous and conforming with the views of Hamel in [31], [32]; and (3) it endows the axiomatization with the threefold interdisciplinary character (mathematical physics, logic, and philosophy of science) stressed in [17].

${ }^{5}$ And, indeed, in my university curriculum I never had a formal training in mathematical logic, nor attended lectures on technical parts of it.

${ }^{6}$ See the paper by H. Hermes and the discussion on it in [22]; see also, there, Apostel's three questions on p. 34, and the replies to them. This attitude is shortly described in parts (b) to (c) of footnote 24 in [5], p. 71: it would be unusual to include modal axioms in physical theories because modal logic is little known [developed] and it is not customary to base such theories on it [Hermes].

${ }^{7}$ From the Preface, p. v, and the Table of Contents, pp. vii to ix, it appears that the proceedings consist of 29 selected papers from symposia and contributed sessions, and only Bressan has more than one. The symposium Modality and the analysis of scientific proposition, Part VIII of the proceedings, consists of five papers: 1) Bressan's conference paper [7],
} 
same degree of appreciation appears from the review by Garson ([27]) of Bressan's [5] paper. ${ }^{8}$ In addition, $(\alpha)$ some advanced versions of Bressan's modal logic have been used by biologists to substantially improve earlier axiomatizations of fundamental biological laws ([45]) or have interested linguists, for instance for the applications of these versions to probability theory $([9])$. ( $\beta$ ) Furthermore, Bressan's (interpreted) logic, that is its semantics, interested an interdisciplinary symposium in 1991 - see [14]; and additional interest in Bressan's semantics is documented in the papers [15], [16], which are strictly related to [14]. $(\gamma)$ Bressan's theory of modal logic is analyzed, sometimes with very interesting contributions, ${ }^{9}$ in papers of pure logic, where its applications to physics are disregarded. Similar considerations apply to Bressan's papers on the logic of senses. ${ }^{10}$

The facts mentioned here support Truesdell's assertions in his 1986 supporting letter, detailed in part (h) of (D) below.

(C) In [48], essay 39, Truesdell presents strongly motivated criticisms against the 'Suppesian' philosophers of science, who are dealing with mechanics (and logic). ${ }^{11}$ These polemic arguments (see Part III of essay 39, especially sections 12 to 15 , pp. 527 to 538) are related to the publication of the Suppesians' paper [37]. Truesdell communicated this paper for publication in spite of Hamel's authoritative and reasonable (initial) strong objections - p. 523, l. -10 to p. 524, l. 9; and he succeeded in carrying it out in order to stimulate discussions on an important foundational subject to which the authors had unsuccessfully tried to contribute. ${ }^{12}$ Truesdell was

pp. 285 to $303 ; 2)$ Suppes' conference paper The essential but implicit role of modal concepts in science, pp. 305 to 314; 3) Bressan's comments on 2), pp. 315 to 321; 4) van Fraassen's comments on 1) and 2), pp. 323 to 330; and 5) Bressan's reply to 4), pp. 331 to 334.

${ }^{8}$ This review, written by a philosopher of science who knows logic, mechanics, and italian, is very fit to quickly obtain some knowledge of the main part of [5]. In it initially Garson says: 'The central contention of Bressan's interesting monograph is that the introduction of ... physical necessity is essential to the development of a clear and precise axiomatization of classical mechanics'; and, in the end, he adds: 'in conclusion, the reviewer believes that this monograph represents an important contribution. Bressan's theory is clearly deeper and more useful than' [37].

${ }^{9}$ See [41], [38], [39], [51], [52], [53]. See also the preface and chapter 2 in [26], [1], who applies Bressan's absolute attributes to various logics, and [28], p. 269 and [29], p. 121.

${ }^{10}$ For instance in [3], [10], [11], [13], [4], and [2].

${ }^{11}$ Besides McKinsey, Sugar, and Suppes himself, Suppesians include Sneed, Moulines, Montague, and Stegmüller - see [48], p. 505, l. -13 ('1. -n' meaning the n-th line from bottom), p. $517,1.11$, and pp. 532 to 535 .

Strangely, Stegmüller (correctly) regards as unreasonable his own interpretation of Carnap's program - see [48], p. 531, ll. -14 to -10 - and he (incorrectly) imputes it to Carnap - p. 506, 1l. 13 to 24. Furthermore he strongly overestimates the (practically nonexistent) contribution of the 'earlier Suppesians' to the purely mathematical aspect of physical theories - p. 506, 1. -3. And similarly behaved Suppes, Moulines, and Sneed about 20 years after the publication of [37], when Suppes had acknowledged that in certain criticisms to this paper Truesdell was right - see [48], p. 528 , ll. -8 to -4 .

${ }^{12}$ At the outset of [37] Truesdell wrote a strongly critical footnote, which was absolutely necessary to justify the publication of that paper in spite of Hamel's objections; these being shared by all practitioners of mechanics that I know who are aware of that paper. 
very correct with the authors of that paper; in particular, he avoided including in his preliminary footnote to it the strong additional criticism later presented in [48], p. 529, ll. 15 to $22{ }^{13}$

(D) Truesdell sent me a confidential copy of his 1986 letter to the Accademia dei Lincei mentioned above. In it Bressan's [12] paper is strongly supported as a starting point for the extension to continuous media of Bressan's [5] particle theory. Here are the main points of Truesdell's letter (in italian).

(a) The subject of this Memoria belongs to Hilbert's sixth problem, 'Mathematische Behandlung der Axiome der Physik ...', which presently is only partially solved.

Truesdell mentions the remarkable contributions given to its solution by Noll, Williams, Gurtin, Appleby, Šilhavý, Ziemer, Matolecsi, among others, then adds:

(b) All of them have used only the procedure of mathematical analysis. Their axioms are proposed as necessary conditions to construct mathematical structures fit for consistent and clear applications to physical systems and present-day technology... .

(c) In 1962 prof. Bressan, considering only mass-point systems, in a deep work of well known difficulty, reached an impeccable solution in the sense of mathematical logic and he was the first to do so.

(d) In his more recent studies he treats classical mechanics in a universe that contains mathematical objects called 'observers', and

(d') he adds a suitable existence axiom that was lacking. ${ }^{14}$

(e) In addition, he constructs a formal concept of physical possibility which abstractly represents a physical experiment. ${ }^{15}$

\footnotetext{
${ }^{13}$ The latter was motivated by the iterated strong overestimation of [37] mentioned in the second paragraph of footnote 11 above. See also [48], pp. 567 to 568, and p. 570, 1. -9, where the self-estimations of the Suppesians mentioned there on p. 517 are extended. By Truesdell's correctness of excluding the additional criticism in the preliminary footnote, only recently Bressan became aware of it and its motivations, and in his (2002) presentation he avowed to share Truesdell's criticism completely. This is relevant for the sense of Truesdell's assertion: 'Of course I do not imply that any of them shares any of my views' written by him in the Errata for [48] after he has given thanks to Bressan and four others.

In (2002) Bressan also emphasized both the nice analysis of physicists' language in [21] $\mathbf{4 1} \mathbf{b}$, p. 168, and the Suppesians' ignorance of this language evidenced in [48], p. 526, ll. 2 to 4; the gravity of this ignorance being seriously increased by the Suppesians' self-overestimations.

${ }^{14}$ The lacking axiom is Ammissione 10.2 in [5], p. 106 (also recalled in [6], footnote 53, p. 110). This is framed as a possibility condition, but (d') is correct because of some peculiar technical features of [5]; and this shows that Truesdell had grasped the main technical semantical features of that paper.

${ }^{15}$ This construction, performed in [5] and refined later in [6] and [8], agrees with the views of [31], [32].
} 
(f) Presently prof. Bressan is completing his logical axiomatization of mechanics with some of his pupils (Montanaro, Pitteri, etc.), and in a way capable of including the mechanics of continuous media in the sense of Noll and his followers.

(g) After a digression Truesdell recommends the publication of Bressan's [12] paper because 'it carries out a remarkable step' in the program mentioned in $(\mathrm{f})$, the main circumstances relevant for it having been sketched in (a) to (e).

(h) Lastly Truesdell adds that Bressan is the unique person (as far as he knows) capable of mastering mathematical logic, rational mechanics, electromagnetism and special relativity; in his opinion Italy and the Accademia dei Lincei can be proud of him.

Here, as well as in his book [48], Truesdell mentions fields outside physics, like logic and philosophy of science. His remarks about Bressan's high standing in these fields get a very authoritative support by earlier assertions of the philosopher of science N. Belnap in his Foreword to GIMC, that is, $[6] .^{16}$

(E) In his [17] conference paper Bressan describes the interdisciplinary character of certain articles à la Mach-Painlevé which belong to mathematical physics, logic, and philosophy of science. ${ }^{17}$ He remarks that this character sometimes strongly contributes to serious misunderstandings of these ar-

\footnotetext{
${ }^{16}$ In his foreword, pp. xiii to xxv of GIMC, Belnap preliminarily says that GIMC 'is the most important contribution to date concerning the introduction of quantifiers into modal logic. It surpasses any article or book in the generality of its conceptions, the degree of their development and the profundity of their analysis' (p. xiii, l. 3). Belnap especially points out Bressan's 'new analysis of predication' (p. xiv, l. 10).

In the subsequent sections 1 to 8 Belnap details and motivates his preliminary assertions. For instance he says (p. xiv, section 1, l. 3): ' $\ldots$ that [Bressan's modal language] $M L^{\nu}$ is - uniquely among modal logics - a complete type theory with no upper limit on its types, is extremely important'. Indeed, as is broadly mentioned on p. xvii, l. -9, by the lack of this limit GIMC solves positively the problem considered in [20], pp. 195 to 196. Among Bressan's 'distinctive semantic features' (pp. xviii to xxiii) Belnap mentions attributes, lambda abstraction, and definite descriptions. Furthermore, in New Directions (p. xxiv, ll. 1 to 6), Belnap especially points out Bressan's notion of absolute attribute, and says: 'The articulation and deployment of this notion is extremely important to Bressan's enterprise ...'. This is confirmed, for instance, by [1]. Lastly, let us note that the citesmanship assertion in [48], p. $532,1 .-12$, is supported by section 16, p. xxiv, 1. -13 in GIMC, where Belnap says that GIMC 'does not itself contain an axiomatization of physics (Bressan has written on this elsewhere in a somewhat different form ...' (he refers to [5], where modalities are phrased in a rather unusual but extensional language).

${ }^{17}$ Indeed, after two unsuccessful extensional (hence non-modal) attempts at rendering [40] rigorous (in [33], [34]), the philosopher of science H. Hermes used some modal operators - see $[22]$ and foonote 6 above. Additional evidence of the interest of philosophers of science for the above interdisciplinarity is given by the pioneering contribution of Burks [19], which was considered in [5], on pp. 57, 72, and 84 (footnotes 6, 25, and 29), thanks to Carnap. Indeed, the notion of physical possibility introduced there (footnotes 4 and 6 above) and refined later (footnote 15) is a special version of Burks' causal possibility.
} 
ticles, involving even their contents. ${ }^{18}$ That such mistakes could happen had been substantially foreseen in the 1959 Paris meeting ([22]), especially by $\mathrm{H}$. Hermes (see footnote 5 above).

In (2002), on the basis of his own observations, Bressan emphasized that the work done, and being done, to render the approach in [40] rigorous and to generalize it is still widely ignored by mathematical physicists. This confirms that Truesdell's merits illustrated above are really very unusual.

\section{References}

[1] J. Bacon. Substance and first-order quantification over individual concepts. J. Symbolic Logic, 45:193-203, 1980.

[2] C. Bonotto. A generalization of the adequacy theorem for the quasi-senses. Notre Dame J. of Formal Logic, 31:560-575, 1990.

[3] C. Bonotto and A. Bressan. On generalized synonymy notions and corresponding quasi-senses. Atti Accad. Naz. Lincei, Cl. Sci. Fis. Mat. Natur. Mem. (8), Mat., 17:163-209, 1984.

[4] C. Bonotto and A. Zanardo. A non-compactness phenomenon in logics with hyperintensional predication. J. Philosophical Logic, 18:383-398, 1989.

[5] Aldo Bressan. Metodo di assiomatizzazione in senso stretto della meccanica classica. applicazione di esso ad alcuni problemi di assiomatizzazione non ancora completamente risolti. Rend. Sem. Mat. Univ. Padova, 32:55-212, 1962.

[6] Aldo Bressan. A general interpreted modal calculus. Yale University Press, New Haven-London, 1972. Foreword by N. D. Belnap, Jr., 327 pages.

[7] Aldo Bressan. On the usefulness of modal logic in the axiomatization of physics. In K.F. Shaffner and R.S. Cohen, editors, Proceedings of the 1972 biennial meeting of the Philosophy of Science Association, Lansing, Michigan, pages 285-303, Dordrecht and Boston, 1974. Reidel Publishing Co.

[8] Aldo Bressan. (a) On physical possibility and (b) Supplement: a much used notion of physical possibility and Gödel's undecidability theorem. In M.L. Dalla Chiara Scabbia, editor, Italian Studies in Philosophy of Science, pages 197-210 and 211-214. North Holland Publishing Co., Amsterdam, 1981.

\footnotetext{
${ }^{18}$ Interdisciplinary articles of this kind are exceptionally rare. Thus their reviewers are likely to be unaware of their mentioned character if they spend little time on introductions and references.
} 
[9] Aldo Bressan. Extensions of the modal calculi $M C^{\nu}$ and $M C^{\infty}$. Comparison of them with similar calculi endowed with different semantics. Application to probability theory. In Uwe Mönnich, editor, Aspects of philosophical logic, some logical forays into central notions of linguistics and philosophy (Synthese Library, vol. 147), pages 21-66. Reidel Publishing Co., Dordrecht, Holland, 1981. Proceedings of a workshop on formal semantics of natural languages held in Tübingen in 1977.

[10] Aldo Bressan. On the interpreted sense calculus $S C_{\alpha}^{\nu}$. In G. Dorn and P. Weingartner, editors, Foundations of Logic and Linguistics: problems and their solutions, pages 427-463, New York and London, 1985. Plenum Publishing Corporation.

[11] Aldo Bressan. On the logic of senses. an anomalous use of belief sentences, its rigorous and formal treatment. In Atti degli Incontri di Logica Matematica (Siena 1985, Padova, 1985, Siena 1986), vol. 3, pages 137-165, Siena, 1987. Università di Siena.

[12] Aldo Bressan. Towards axiomatic foundations of the Mach-Painlevé type for classical or relativistic theories of continuous media. Atti Accad. Naz. Lincei, Cl. Sci. Fis. Mat. Natur. Mem. (8), Fis., 19:1-21, 1987.

[13] Aldo Bressan. New semantics for the extensional but hyper-intensional part $L_{\alpha}$ of the modal sense language $S L_{\alpha}$. Notre Dame J. of Formal Logic, 32:47-86, 1991.

[14] Aldo Bressan. A semantical theory connected with space-time. In U. Mayer and H.J. Schmidt, editors, Proceedings of the Symposium 'Semantical aspects of space-time theories', Bielefeld, 1991, pages 53-65, Leipzig, 1994. Wissenschaftsverlag.

[15] Aldo Bressan. Again on relativistic semantics. Logic and logical philosophy, 3:23-36, 1995.

[16] Aldo Bressan. Some answers to Max Urchs' comments to a paper of A. Bressan. Logic and logical philosophy, 4:123-131, 1996.

[17] Aldo Bressan. On the interdisciplinary character of rigorous articles à la Mach-Painlevé (in italian). Conference presented at the Convegno in onore dei 90 anni del prof. Giuseppe Grioli, Padova, Accademia Patavina, April $3-4,2002$.

[18] B. Budiansky and Lev Truskinovsky. On the mechanics of stress-induced phase transformations in zirconia. J. Mech. Phys. Solids, 41:1445-1459, 1993.

[19] A.W. Burks. The logic of causal propositions. Mind, 60:363-383, 1951.

[20] R. Carnap. Meaning and necessity. The University of Chicago Press, Chicago, 1956. 1st version 1947. 
[21] R. Carnap. Introduction to symbolic logic. Dover Publications, Inc., New York, 1958. German edition 1954.

[22] J.L. Destouches. La méthode axiomatique dans les mécaniques classiques et nouvelles. Gauthier-Villars, Paris, 1963. Actes du 4me Colloque International de Logique et Philosophie des Sciences, Institut Henri Poincaré, Paris, 1959, subventionée par l'Union Internationale d'Histoire et de Philosophie des Sciences.

[23] Jerald L. Ericksen. Local bifurcation theory for thermoelastic Bravais lattices. In J.L. Ericksen, R.D. James, D. Kinderlehrer, and M. Luskin, editors, Microstructure and phase transition, Vol. 54 of the IMA Volumes in Mathematics and its Applications. Springer-Verlag, New York, etc., 1993.

[24] Jerald L. Ericksen. On the theory of the $\alpha-\beta$ phase transition in quartz. J. of Elasticity, 63:61-86, 2001.

[25] G. Fadda, L. Truskinovsky, and G. Zanzotto. Unified Landau description of the tetragonal, orthorhombic, and monoclinic phases of zirconia. Phys. Rev. B, 66:174107 1-10, 2002.

[26] D. Gallin. Intensional and higher-order modal logic. North-Holland Publishing Company, Paris, 1975. The book is written in memory of $R$. Montague, and has the subtitle With application to Montague semantics.

[27] J. Garson. Review of A. Bressan's 'Metodo di assiomatizzazione...'. J. of Symbolic Logic, 38:144-145, 1973.

[28] J. Garson. Quantification in modal logic. In Gabbay and Günthner, editors, Handbuch of Philosophical Logic, vol. 2, pages 249-307. ??, ??, 1984.

[29] J. Garson. Completeness of some quantified modal logics. In K. Lambert, editor, Philosophical Applications of Free Logic, pages 111-142. Oxford University Press, Oxford, New York, 1991.

[30] M. Golubitsky, D.G. Schaeffer, and I.N. Stewart. Singularities and groups in bifurcation theory, Vol II. Springer Verlag, New York, etc., 1988. Applied Mathematical Sciences vol.69.

[31] G. Hamel. Über die Grundlagen der Mechanik. Mathematische Annalen, 66:350-397, 1908.

[32] G. Hamel. Die Axiome der Mechanik, volume 5 of Handbuch der Physik, pages 1-42. Springer-Verlag, Berlin, 1927.

[33] H. Hermes. Eine Axiomatisierung der Allgemeinen Mechanik. Verlag von Hirzel, Leipzig, 1938. Forschungen zur Logik und zur Grundlegung der exacten Wissenschaften, vol. 3. 
[34] H. Hermes. Zur Axiomatisierung der Mechanik. In F. Bethuel, G. Huisken, S. Müller, K. Steffen, S. Hildebrandt, and M. Struwe, editors, Proceedings of an International Symposium on Axiomatic Method held at Berkeley, 195\%1958, page 212, Amsterdam, 1959. North Holland Publishing Co.

[35] International Tables. International Tables for X-ray Crystallography, Volume A. Reidel Publishing Company, Dordrecht, Boston, 1996. T. Hahn (Ed.).

[36] Richard D. James. The stability and metastability of quartz. In Stuart S. Antman, Jerald L. Ericksen, David Kinderlehrer, and Ingo Müller, editors, Metastability and Incompletely Posed Problems, Vol. 3 of the IMA Volumes in Mathematics and its Applications. Springer-Verlag, New York, etc., 1987.

[37] J.C.C. McKinsey, A.C. Sugar, and P. Suppes. Axiomatic foundations of classical particle mechanics. J. of Rat. Mech. Anal., 2:253-272, 1953.

[38] Eugenio G. Omodeo. The elimination of descriptions from A. Bressan's modal language $M L^{\nu}$ on which the logical calculus $M C^{\nu}$ is based. Rend. Sem. Mat. Univ. Padova, 56:269-292, 1977.

[39] Eugenio G. Omodeo. Three existence principles in a modal calculus without descriptions contained in A. Bressan's $M C^{\nu}$. Notre Dame J. of Formal Logic, 21:711-727, 1980.

[40] Paul Painlevé. Les axiomes de la mécanique. Gauthier-Villars, Paris, 1922.

[41] Z. Parks. Investigations into quantified modal logic - I. Studia Logica, 35:109-125, 1976.

[42] Mario Pitteri. On the axiomatic foundations of temperature. In Appendix G6 of C. Truesdell's Rational Thermodynamics, 2nd Edition, pages 522544. Springer-Verlag, New York, etc., 1984.

[43] Mario Pitteri. On bifurcations in multilattices. In R. Monaco, M. Pandolfi, and S. Rionero, editors, Proceedings of WASCOM 2001, Porto Ercole, Italy, pages 397-408, Singapore etc., 2002. World Scientific.

[44] Mario Pitteri and Giovanni Zanzotto. Continuum models for phase transitions and twinning in crystals. CRC/Chapman \& Hall, Boca Raton, London, etc., 2002.

[45] Martino Rizzotti and Alberto Zanardo. Assiomatizzazioni della genetica. Aspetti non estensionali in alcune leggi elementari della genetica classica. Atti Accad. Naz. Lincei, Cl. Sci. Fis. Mat. Natur. Mem. (9), Mat. Appl., 2:87-125, 1993. 
[46] Narendra K. Simha and Lev Truskinovsky. Phase diagram of zirconia in stress space. In R.C. Batra and M.F. Beatty, editors, Contemporary research in the mechanics and mathematics of materials. CIMNE, Barcelona, 1996.

[47] Pierre Tolédano and Vladimir Dmitriev. Reconstructive phase transformations: in crystals and quasicrystals. World Scientific, Singapore etc., 1996.

[48] Clifford A. Truesdell. An idiot's fugitive essays on science: methods, criticism, training, circumstances. Springer Verlag, New York, etc., 1984.

[49] Clifford A. Truesdell. Rational thermodynamics, second edition. Springer Verlag, New York, etc., 1984.

[50] Clifford A. Truesdell and R. G. Muncaster. Fundamentals of Maxwell's kinetic theory of a simple monatomic gas, treated as a branch of Rational Mechanics. Academic Press, New York, etc., 1980.

[51] Alberto Zanardo. A completeness theorem for the general interpreted modal calculus $M C^{\nu}$ of A. Bressan. Rend. Sem. Mat. Univ. Padova, 64:39$57,1981$.

[52] Alberto Zanardo. On the equivalence between the calculi $M C^{\nu}$ and $E C^{\nu+1}$ of A. Bressan. Notre Dame J. of Formal Logic, 24:367-388, 1983.

[53] Alberto Zanardo. Individual concepts as propositional variables in $M L^{\nu+1}$. Notre Dame J. of Formal Logic, 25:332-346, 1984. 